\title{
Photothermal optical coherence tomography for investigation and imaging photothermal trapping of gold nano-rods in clear media and biological tissue
}

\author{
Yong Hu*, Adrian Podoleanu, ANd George Dobre \\ Applied Optics Group, School of Physical Sciences, University of Kent, CT2 7NH Canterbury, UK \\ *y.hu-240@kent.ac.uk
}

\begin{abstract}
A quantitative spectrometer-based photothermal optical coherence tomography (PT-OCT) system is employed to investigate and image the photothermal trapping of gold nano-rods (GNRs) in clear and biological media. The PT-OCT system is calibrated through dynamic phase measurements of piezo motion with known driving parameters. We measure and compare the displacement sensitivities of the PT-OCT system at different camera exposure time settings in two configurations: with a distinct reference path; and with a common path. The displacement sensitivity of the system in the common path configuration is improved from $1.5 \mathrm{~nm}$ to $0.17 \mathrm{~nm}$ by performing Fourier analysis on the output phase. The minimum Ti:Sa power capable of inducing a detectable photothermal response of GNRs is measured to be $0.5 \mathrm{~mW}$. This value agrees with the latest reported minimum Ti:Sa power for photothermal trapping GNRs. The PT-OCT system is used to generate en-face images of photothermal trapped GNRs in the water solution and in the biological tissue. By displaying the difference between successive en-face phase images, spatial distribution patterns of the aggregated GNRs, resulted from the photothermal trapping, are clearly outlined with great contrast. The photothermal trapping of GNRs in tissue shows a greater complexity than in the clear media. The limitation of the PT-OCT technology is discussed. The study proves the potential of PT-OCT for imaging the photothermal trapping of GNRs.
\end{abstract}

\section{References and links}

1. I. A. Wani, "Biomedical Applications of Gold Nanoparticles: Recent Advances and Future Prospects," in Biomedical Engineering: Concepts, Methodologies, Tools, and Applications, IRMA, (IGI Global, 2017).

2. Particle Works white paper, "Gold Nanoparticles," http://www.particle-works.com/product-category/gold-nanoparticles/?gclid=CObuMKB79ECFcaV7QodvjEI1A.

3. F. Hajizadeh, and S.Nader S.Reihani, "Optimized optical trapping of gold nanoparticles," Opt. Express 18(2), 551-559 (2010).

4. L. Tong, Q. Wei, Al. Wei, and J. X. Cheng, "Gold nanorods as contrast agents for biological imaging: optical properties, surface conjugation and photothermal effects," Photochem Photobiol. 85(1), 21 (2010).

5. J. Stone, St. Jackson, and D. Wright, "Biological applications of gold nanorods," NanoMed. and NanoBio. 3(1), 100 (2010).

6. Ch. Schoen, "Gold Nanostructures: Properties and Applications," http://www.sigmaaldrich.com/technical-documents/articles/materialsscience/nanomaterials/gold-nanostructures.html

7. H. Chen, E. Gratton, and M. A. Digman, "Self-assisted optothermal trapping of gold nanorods under two-photon excitation," Methods Appl. Fluoresc. 4(3), 035003 (2016).

8. S. J. Oldenburg, J. B. Jackson, S. L. Westcott, and N. J. Halas, "Infrared extinction properties of gold nanoshells," Appl. Phys. Lett. 75(19), 2897-2899 (1999).

9. J. R. Cole, N. A. Mirin, M. W. Knight, G. P. Goodrich, and N. J. Halas, "Photothermal efficiencies of nanoshells and nanorods for clinical therapeutic applications," J. Phys. Chem. C 113(28), 12090-12094 (2009).

10. Ch. H. Chou, Ch. D. Chen, and C. R. Ch. Wang, "Highly Efficient, Wavelength-Tunable, Gold Nanoparticle Based Optothermal Nanoconvertors," J. Phys. Chem. B 109(22), 11135-11138 (2005).

11. T. Niidome, M. Yamagata, Y. Okamoto, Y. Akiyama, H. Takahashi, T. Kawano, Y. Katayama, and Y. Niidome, "PEG-modified gold nanorods with a stealth character for in vivo applications," J Control Release 114(3), 343-347(2006).

12. D. Braun and A. Libchaber, "Trapping of DNA by Thermophoretic Depletion and Convection," Phys. Rev. Lett. 89, 188103 (2002).

13. Y. Liu and A. W. Poon, "Flow-assisted single-beam optothermal manipulation of microparticles," Optics Express 18(17), 18483-18491(2010).

14. H. Chen, E. Gratton, and M. A. Digman, "Spectral properties and dynamics of gold nanorods revealed by EMCCD-based spectral phasor method," Microsc. Res. Tech. 78(4), 283-293 (2015).

15. K. Imura, T. Nagahara, and H. Okamoto "Near-field two-photon-induced photoluminescence from single gold nanorods and imaging of plasmon modes," J. Phys. Chem. B 109(27), 13214-13220 (2005).

16. D. Huang, E. A. Swanson, C. P. Lin, J. S. Schuman, W. G. Stinson, W. Chang, M. R. Hee, T. Flotte, K. Gregory, C. A. Puliafito, and J. G. Fujimoto, "Optical Coherence Tomography," Science 254, 1178-1181 (1991).

17. A. Gh. Podoleanu, G. M. Dobre, D. A. Jackson, "En-face coherence imaging using galvanometer scanner modulation" Opt. Letters 23, 147-149 (1998).

18. A. Gh. Podoleanu, "Optical Coherence Tomography," Journal of Microscopy 247(3), 209-299 (2012).

19. D. C. Adler, R. Huber, and J. G. Fujimoto, "Phase sensitive optical coherence tomography at up to 370,000 lines per second using buffered Fourier domain mode-locked lasers," Opt. Letters 32(6), 626-628 (2007). 
20. M. Sticker, M. Pircher, E. Gotzinger, H. Sattmann, A. F. Fercher, and C.K. Hitzenberger, "En face imaging of single cell layers by differential phase-contrast optical coherence microscopy" Opt. Lett. 27(13), 1126-1128 (2002).

21. Z. Chen, T. E. Milner, D. Dave, and J. S. Nelson, "Optical Doppler tomographic imaging of fluid flow velocity in highly scattering media," Opt. Lett. 22(1), 64-66 (1997).

22. R. K. Wang, and A. L. Nuttall, "Phase sensitive optical coherence tomography imaging of the tissue motion within the organ of Corti at a sub-nanometre scale a preliminary study," J. Biomed Opt. 15(5), 056005 (2010).

23. R. K. Manapuram, V. G. R. Manne, and K. V. Larin, "Phase-sensitive swept source optical coherence tomography for imaging and quantifying of microbubbles in clear and scattering media," Journal of Applied Physics 105(10), 102040 (2009).

24. Ch. Joo, T. Akkin, B. Cense, B. H. Park, and J. F. de Boer, "Spectral-domain optical coherence phase microscopy for quantitative phase-contrast imaging," Opt. Lett. 30(16), 2131-2133, (2005).

25. M. V. Sarunic, S. Weinberg, and J. A. Izatt, "Full-field swept-source phase microscopy," Opt. Lett. 31(10), 1462-1464 (2006).

26. M. A. Choma, A. K. Ellerbee,S. Yazdanfar, and J. A. Izatt, "Doppler flow imaging of cytoplasmic streaming using spectral domain phase microscopy," J. Biomed. Opt. 11(2), 024014 (2006).

27. Ch. Joo, T. Akkin, Ki H. Kim, and J. E. de Boer, "Spectral-domain optical coherence phase and multiphoton microscopy," Opt. Lett. 32(6), 623-625 (2007).

28. D. C. Adler, Sh. W. Huang, R. Huber, and J. G. Fujimoto, "Photothermal detection of gold nanoparticles using phase-sensitive optical coherence tomography," Opt. Express 16(7), 4376-4393 (2008).

29. M. C. Skala, M. J. Crow, A. Wax, and J. A. Izatt, "Photothermal Optical Coherence Tomography of Epidermal Growth Factor Receptor in Live Cells Using Immunotargeted Gold Nanospheres," Nano Lett. 8(10), 3461-3467 (2008).

30. J. M. Tucker-Schwartz, T. A. Meyer, C. A. Patil, C. L. Duvall, and M. C. Skala, "In vivo photothermal optical coherence tomography of gold nanorod contrast agents," Opt. Express 3(11), 2881-2895 (2012).

31. D. Boyer, P. Tamarat, A. Maali, B. Lounis, and M. Orrit, "Photothermal imaging of nanometre-sized metal particles among scatterers," Science 297(5584), $1160-1163$ (2002).

32. J. Kim, J. Oh, and T. E. Milner, "Measurement of optical path length change following pulsed laser irradiation using differential phase optical coherence tomography," J. Biomed. Opt. 11(4), 041122 (2006).

33. Y. Jung, R. Reif, Y. Zeng, and R. K. Wang, “Three-Dimensional High-Resolution Imaging of Gold Nanorods Uptake in Sentinel Lymph Nodes," Nano Lett. 11(7), 2938-2943 (2011).

34. J. H. Pai, T. Liu, H. Y. Hsu, A. B. Wedding, B. Thierry, and P. O. Bagnaninchi, "Molecular photo-thermal optical coherence phase microscopy using gold nanorods," RSC Advances 4(51), 27067-27073(2014)

35. Z. Yaqoob, W. Choi, S. Oh, N. Lue, Y. Park, Ch. F. Yen, R. R. Dasari, K. Badizadegan, and M. S. Feld, "Improved phase sensitivity in spectral domain phase microscopy using line-field illumination and self phase-referencing," Opt. Express 17(13), 10681-10687 (2009).

36. B. H. Park, M. C. Pierce, B. Cense, S. H. Yun, M. Mujat, G. J. Tearney, B. E. Bouma, and J. F. de Boer, "Real-time fiber-based multi-functional spectraldomain optical coherence tomography at 1.3 $\mu \mathrm{m}$,” Opt. Express 13(11), 3931-3944 (2005).

37. B. F. Kennedy, S. H. Koh, R. A. McLaughlin, K. M. Kennedy, P. R. T. Munro, and D. D. Sampson, "Strain estimation in phase-sensitive optical coherence elastography," Opt. Express 3(8), 1865-1879 (2012).

38. B. J. Vakoc, S. H. Yun, J. F. de Boer, G. J. Tearney, and B. E. Bouma, "Phase resolved optical frequency domain imaging," Opt. Express 13(14), 5483-5493 (2005).

39. R. V. Kuranov, A. B. McElroy, N. Kemp, S. Baranov, J. Taber, M. D. Feldman, and Th. E. MilnerGas, "Cell Referenced Swept Source Phase Sensitive Optical Coherence Tomography," IEEE Photonics Technology Letters 22(20), 1524-1526 (2010).

\section{Introduction}

During the last 20 years, gold nanoparticles (GNPs) have been extensively used as contrast agents in biomedical imaging and therapy agents in photothermal therapeutics [1]. The application of GNPs is based on the fact that they exhibit useful optical and photothermal properties which can be tuned to absorb and scatter light across the visible and NIR region [2]. GNPs are outstanding candidates for optical tweezer based micromanipulation inside living cells, and the optical trapping efficiency of a single GNP is 7 fold better than a similarly sized latex particle [3]. GNPs can also convert optical energy into heat, endowing them with intense agents for cancer treatments [4]. These optical and photothermal properties of GNPs result from the collective waves that travel along the metal/liquid interface and are heavily determined by precise control over the particles' shape, size or architecture [5]. The earliest developed GNPs, gold nano-spheres, were not usually optimized for biomedical applications due to their limited peak absorption in the transmission window $(650-900 \mathrm{~nm})$ for biological entities [6]. By contrast, other nano-structures, like gold nanoshells (GNSHs) and gold nano-rods (GNRs), exhibit a tunable peak absorption wavelength in a wider electromagnetic range. For instance, the peak absorption wavelength of GNRs can be tuned from $600 \mathrm{~nm}$ to $1400 \mathrm{~nm}$ to cover the visible and NIR regions by varying their aspect ratio [7]. The peak absorption wavelength of GNSHs can be tuned from $800 \mathrm{~nm}$ to $2200 \mathrm{~nm}$ by varying the ratio of core radius to gold shell thickness [8]. Compared with GNSHs, GNRs have several advantages for in vivo molecular imaging [9]. Firstly, due to their smaller size (tens of nanometres), GNRs are more mobile in thin blood vessels, whereas GNSHs are hundreds of nanometres in size. Secondly, the absorption peak of GNRs is much narrower than that of GNSHs, which is desirable to decrease the attenuation of the imaging beam by the contrast agent. Thirdly, compared with GNSHs, GNRs find wider use in photothermal therapeutics since they have higher photothermal conversion efficiencies in the NIR region than GNSHs [10,11], which gives them a unique photothermal trapping effect [7]. The photothermal trapping of GNRs happens when they are exposed to a tightly focused NIR laser beam. In the case, GNRs absorb and then convert the laser energy into heat efficiently. The 
created heat generates a temperature gradient in the media. As a result, the temperature gradient creates thermophoresis and convection flow in the media, which work together to trap and redistribute surrounding GNRs [7,12]. The theoretical and experimental studies of photothermal trapping of other micro particles have been explained previously [12,13], in which the photothermal trapping is proved to be an alternative approach to the optical trapping of micro particles. However, only a few reports on photothermal trapping of GNRs were published until recently [7,14]. In [7], the photothermal trapping process of GNRs was investigated and imaged by detecting a two-photon luminescence (TPL) signal. GNRs radiate TPL, as a unique nonlinear optical property, when they are exposed to a Ti:Sa laser beam [15]. Moreover, the process of photon absorption using a tightly focused beam with a depth of focus of the order of several microns (high NA) results in the localised heating in a small volume overlapping with the focal spot region near a glass surface. The heat spots around GNRs were shown to contribute to the trapping of other particles, as well as making GNRs subject to trapping themselves. Because $96 \%$ of the photon energy absorbed by GNRs is converted into heat via non-radiative electron relaxation [7], the resulting TPL signal was weak, and TPL detection could only be demonstrated for GNRs diffused in the deionized (DI) water, not in biological tissue or other scattering media. The resulting images of GNR aggregation patterns and geometries are therefore specific to a high NA, tightly focused excitation geometry. However, in order to effectively image the photothermal trapping effect of GNRs in scattering media, with a comparatively low NA and longer depth of focus required for deeper penetration into structures, it is advantageous to explore an alternative detection technology, hence this study.

Optical Coherence Tomography (OCT), especially its subsidiary Fourier domain OCT (FD-OCT) is a non-invasive optical diagnostic technique capable of providing depth-resolved images with a micrometre scale resolution to depths of several millimetres of the studied tissue [16-18]. FD-OCT can be implemented in two ways: spectral domain OCT (SD-OCT) employing a broadband light source and a spectrometer; or swept source OCT (SS-OCT) using a swept-laser source and a photodetector. Tiny optical path difference (OPD) variations, of the order of fractions of the axial resolution, can be measured by retrieving the interferometric phase in phase sensitive OCT. Employing phase sensitive OCT, the measured absolute phase value has been used for en-face imaging [19,20]. The phase difference between successive A-scans has been used for Doppler flow OCT [21]. So far, phase sensitive OCT has been reported in a variety of applications, such as evaluating mammalian cochlea motion at sub-nanometre scale [22], imaging and quantifying microbubbles in clear and scattering media with an accuracy of $10 \mathrm{~nm}$ [23], lateral profiling of cells with sub-nanometre sensitivity [24], and cross-sectional imaging of dynamic contractile motion of beating cardiomyocytes at a line rate of $19 \mathrm{kHz}$ [20]. Determined by the implementation, phase sensitive SS-OCT exhibits nanometre scale displacement sensitivity [25], whereas phase sensitive SD-OCT reaches even sub-nanometre scale displacement sensitivity [26,27].

As a functional extension of phase sensitive OCT, photothermal OCT (PT-OCT) has been applied for photothermal detection of GNSHs in clear media [28-30]. PT-OCT is able to identify and separate the GNSHs from surrounding media through the detection of photothermal heating [31], where the photon absorption by GNPs leads to a temperature change in the surrounding environment, causing the thermo-elastic expansion of the media and the shifts in the values of the local refractive index [32]. The local geometric and refractive index changes alter the local optical path length (OPL) that can be directly detected by a phase sensitive OCT system. As a biomedical agent with great potential, GNRs have been used as contrast agents in PT-OCT for in vivo imaging and 3D imaging of tissue structure [30,33], and even for molecular imaging [34]. In these reports, however, the photothermal trapping effect of GNRs to Ti:Sa laser beams was discarded, and the photothermal trapping induced movements of GNRs in the tissue sample was ignored. Although GNRs have been proved as appealing agents in biomedical application, the study of their photothermal trapping effect, especially in biological tissue, is still inadequate. Since $96 \%$ of absorbed photon energy by GNRs is converted into heat [7], we anticipate that PT-OCT may offer an efficiency advantage in the study of photothermal trapping of GNRs compared to the reported TPL detection.

In this paper, we present a theoretical analysis and experimental verification of the phase measurement capability of a phase sensitive SD-OCT system. We detail the methodology to test and quantify the performance of the system, including the method to calibrate the system and the methods to measure the sensitivity and the sensitivity decay of two set-up configurations: one using separate interferometer arms; and the other one employing a common path configuration. We chose the common path system for its superior sensitivity to measure the dependence of the photothermal response of GNRs (diffused in DI water) to both the modulation frequency and the average power of the Ti:Sa laser beam. After these measurements, we observed the aggregated GNRs as a result of the photothermal trapping. In order to image the aggregated GNRs, we performed photothermal imaging instead of detecting the TPL signal. It is concluded that the photothermal imaging is an alternative method to study the photothermal trapping of GNRs. We then injected the GNR solution into a piece of pork tissue and performed photothermal detection to locate GNRs. After the GNRs were located in the tissue sample, we performed photothermal imaging of these GNRs at the allocated depth to monitor their photothermal response. We found that the photothermal trapping of GNRs diffused in biological tissue was 
affected by the tissue structure in a random way, and the photothermal trapping of GNRs in tissue was significantly more complex than that in the DI water.

\section{Theory}

The measurement of OPD fluctuations using phase sensitive OCT has been discussed and mathematically evaluated in earlier studies $[19,22,23,28]$. For FD-OCT, the detected interference term of the spectrum is expressed as $I(k)=2\left|E_{R} E_{S}\right| \cos (2 k z+\varphi)$, where $k=2 \pi / \lambda$ is the wavenumber, $\varphi$ is the random system noise added to phase, and $2 z$ is the OPD between reference and sample paths if OPD $=0$ is placed at the top of the sample. To obtain the depth resolved structural information, coded in magnitude $A(z)$ and phase $\Phi(z)$, of the sample, a complex Fourier transform (FT) of $I(k)$ gives the interference signal $I(z)$ in the time domain:

$$
I(z)=F T[I(k)]=A(z) \exp [i \Phi(z)]
$$

where the magnitude $A(z)$ serves to reconstruct conventional OCT images, and $\Phi(z)$ provides the phase information at the optical depth $z$ in the sample, which is varied as the axial OPD change $z(t)$ over time and can be expressed as:

$$
\Phi(z, t)=2 \times \frac{2 \pi}{\lambda} \times n \cdot z(t)+\varphi
$$

where $\varphi$ is the random noise and the factor of 2 comes from the double path of the beam in the OCT sample arm. For periodic fluctuations, assuming the $z(t)$ variation is created by a sinusoidal vibrating source with vibrating magnitude $A$ and frequency $f_{0}$, Eq. (2) becomes:

$$
\Phi(z, t)=2 \times \frac{2 \pi}{\lambda} \times n \cdot A \cdot \sin \left(2 \pi f_{0} t\right)+\varphi
$$

Since the data detecting rate is much faster than the fluctuation of the noise, by calculating the phase difference $\Delta \Phi(z, t)$ between two successive A-scans, temporally separated by $\Delta t$, the noise term $\varphi$ can be eliminated:

$$
\Delta \Phi(z, t)=\frac{8 \pi^{2}}{\lambda} \times n \cdot A \cdot f_{0} \cdot \cos \left(2 \pi f_{0} t\right) \cdot \Delta t
$$

According to Eq. (3) and Eq. (4), if the vibrating frequency $f_{0}$ is given, by measuring either $\Phi(z, t)$ or $\Delta \Phi(z, t)$, the vibration magnitude $A$ can be obtained. If Eq. (3) is used, the continuity of the $\Phi(z, t)$ trace would be disturbed by the existence of the random noise term $\varphi$, as we observed during the measuring process. If Eq. (4) is used, when the amplitude of $\Delta \Phi(z, t)$ is as low as its noise floor, the $\Delta \Phi(z, t)$ measurement meets its sensitivity limit [22]. To overcome this limit, a fast Fourier transform (FFT) to Eq. (4) gives the amplitude $\left|\mathrm{p}\left(f_{0}\right)\right|$ of the FFT peak at the frequency $f_{0}$, which also yields the vibrating amplitude $A$ :

$$
\operatorname{FFT}[\Delta \Phi(z, t)]=\mathrm{p}(\mathrm{z}, f)=\frac{4 \pi^{2}}{\lambda} \cdot A \cdot f_{0} \cdot \Delta t \stackrel{\text { yields }}{\longrightarrow} A=\frac{\left|\mathrm{p}\left(f_{0}\right)\right| \cdot \lambda}{4 \pi^{2} \cdot f_{0} \cdot \Delta t}
$$

where $f$ is the frequency variable in the frequency domain. Thus, Eq. (3), Eq. (4) and Eq. (5) can be used independently to obtain the vibration magnitude $A$ of a sinusoidal vibration. In this study, the measurements were made on vibrations generated by a piezo actuator. The results obtained from Eq. (3), Eq. (4) and Eq. (5) were compared with each other and then with the data sheet of the actuator to evaluate which equation matched most closely the actuator response. Our phase sensitive OCT system was calibration by the comparison.

\section{Methods}

\subsection{System configuration}

A phase sensitive SD-OCT system was developed as presented in Fig. 1. The OCT source is a superluminescent diode (Amonics, ALS-1050-S, $13 \mathrm{~mW}, 35 \mathrm{~nm}$ bandwidth @ $1050 \mathrm{~nm}$ ). The output light travels through a fibre isolator and subsequently, a $2 \times 2$ fibre-optic Michelson interferometer composed of a 50:50 fibre coupler (Thorlabs, TW1064R5A2A). With collimating lenses, the fibre coupler outputs $2.4 \mathrm{~mm}$ diameter beams in two arms. In the sample arm, after a dichroic mirror, the OCT beam is co-axially combined with a Ti:Sa laser (Coherent, Mira Seed, $3 \mathrm{~mW}$ output power, $80 \mathrm{MHz}$ repetition rate, $2 \mathrm{~mm}$ diameter, $150 \mathrm{fs}$ pulse duration, $25 \mathrm{~nm}$ bandwidth @ $800 \mathrm{~nm}$ ) that acts as the excitation source. The superposed beams are incident on a pair of galvanometer mirrors (Thorlabs, GVS302) and subsequently travel through an objective/scan lens (Thorlabs, LSM02-BB, $f=18$ 
$\mathrm{mm}$ ) producing a focused spot size of $3.1 \mu \mathrm{m}$ on the sample. The Ti:Sa beam is modulated by an optical chopper wheel. In the reference arm, a glass block compensates the dispersion created by the objective lens in the sample arm. Light beams returning from both arms interfere at a commercial spectrometer consisting of a high-speed line scan CMOS camera (Goodrich SUI, SU1024$\mathrm{LDH}$ ) with a maximum line rate of $47 \mathrm{kHz}$ and the digital output rate of 14-bit. The camera has 1024 elements with a pixel size of $25 \times 500 \mu \mathrm{m}$ and a spectral range of $900 \mathrm{~nm}$, resulting in a spectral resolution of $0.088 \mathrm{~nm}$ at the centre wavelength of $1050 \mathrm{~nm}$. The camera output is then digitized by a camera link image acquisition card (National Instruments, PCIe-1430) installed in a computer.

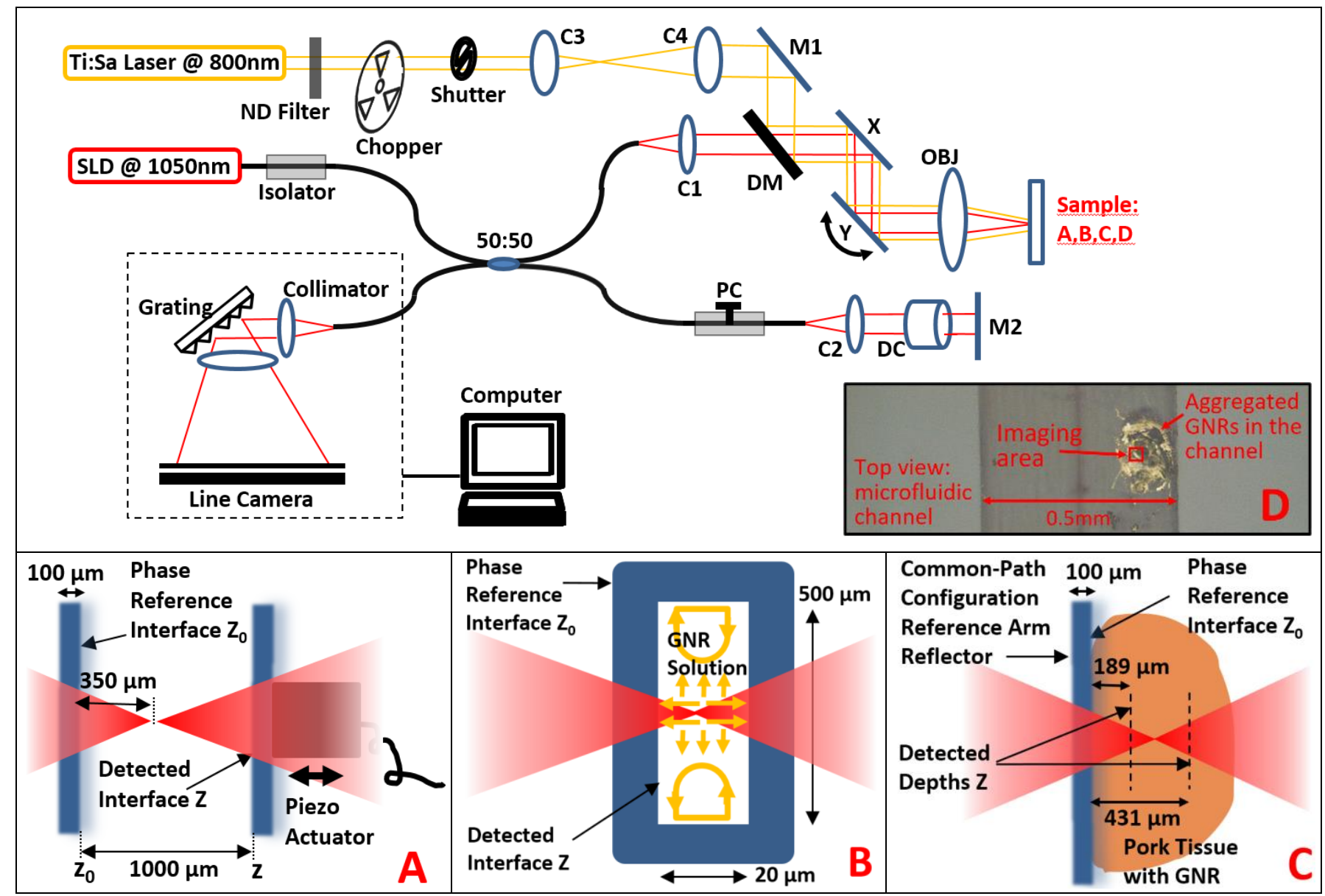

Fig. 1. Schematic layout of phase sensitive SD-OCT with photothermal detection capability. Superluminescent diode (SLD), neutral density filter (ND Filter), collimating lenses (C1,C2), fibre coupler (50:50), achromatic doublets (C3,C4), dichroic mirror (DM), scanning mirrors $(\mathrm{X}, \mathrm{Y})$, objective lens $(\mathrm{OBJ})$, dispersion compensating block (DC), mirrors (M1,M2), polarization controller (PC). The three categories of the sample are shown in inserts (A), (B) and (C). Inset (B) is a cross-sectional view of a glass made microfluidic channel (500 $\mu \mathrm{m}$ wide and 20 $\mu \mathrm{m}$ deep). The surface facing the shooting beam (from left to right) is the top surface of the channel, and the longitudinal axis of the channel is perpendicular to the paper page. Inset (D) shows a $10 \times$ magnified microscopy image of the top view of the front surface of this microfluidic channel $(500 \mu \mathrm{m}$ wide). As seen, in the channel, the aggregation of GNRs (the golden blob in the image) results from the photothermal trapping of Ti:Sa beam.

As the interference light projecting on the pixels on both edges of the camera has no interferometric features, 674 out of 1024 elements in the middle of the camera are enabled. The computer employs a B-spline interpolation algorithm (developed by the authors) to rescale and resample the interference signal and generates an evenly distributed 674-points channelled spectrum in the $k$ (wavenumber)-space. The channelled spectrum then undergoes a complex FFT, obtaining depth-resolved 337-pixel phase and intensity profiles of the target. Measuring the full width at half maximum (FWHM) of A-scan peaks in the intensity profile, the 
OCT system has an axial resolution of $17 \mu \mathrm{m}$ in the air. The imaging depth of $z=1.44 \mathrm{~mm}$ in the air is evaluated, where the sensitivity decays by $6 \mathrm{~dB}$. Using a refractive index $n=1.38$ for pork tissue, the axial resolution and imaging depth in such tissue become respectively $12 \mu \mathrm{m}$ and $1.01 \mathrm{~mm}$. The absolute phase value $\varphi(z, t)$ at a depth of interests $z$ in the sample is retrieved in two steps. Firstly, in the A-scan intensity profile, we identify the pixel index of the interested depth $z$ from its corresponding peak. Secondly, in the A-scan phase profile, we extract the phase value $\varphi(z, t)$ of that pre-identified pixel index.

For an OCT system, the use of a separate reference arm is subject to random phase noise due to mechanical or thermal fluctuations in each independent arm [35]. In this work, as seen in Fig. 1.A, to reduce the dependence on such fluctuations, a 100 $\mu \mathrm{m}$ microscope coverslip is placed at $z_{0}$ in front of the actuated coverslip to provide a reference phase $\varphi\left(z_{0}, t\right)$. Since the phase of all points along the optical beam is detected in the same A-scan, the phase values at all points are affected by the same noise. Consequently, the phase noise can be removed by subtracting the reference phase $\varphi\left(z_{0}, t\right)$ from the phase of interests $\varphi(z, t)$, and the dynamic difference $\Phi(z, t)$ can be expressed as:

$$
\Phi(z, t)=\varphi(z, t)-\varphi\left(z_{0}, t\right)
$$

In practice, $\Phi(z, t)$ is obtained by calculating the cross-power $Y_{z z_{0}}(t)$ of signals from interfaces $z_{0}$ and $z$ :

$$
Y_{z z_{0}}(t)=Y_{\mathrm{z}}(t) \cdot \overline{Y_{z_{0}}(t)}=A A_{0} e^{i\left(\varphi(z, t)-\varphi\left(z_{0}, t\right)\right)}=A A_{0} e^{i(\Phi(z, t))}
$$

where $Y_{z}(t)$ and $Y_{z_{0}}(t)$ are complex values retrieved from the interested interface $z$ and the reference interface $z_{0}$. For the rest of the paper, the expression "detected phase signal" refers to $\Phi(z, t)$ in Eq.(7).

\subsection{Sample preparation}

As seen in Fig. 1.A, the sample employed to calibrate the system is a $100 \mu \mathrm{m}$ glass coverslip that is mounted on a piezo actuator (Thorlabs, PA4FEW). When driven by a pre-set sinusoidal voltage, the piezo actuator reacts with sinusoidal vibration with known frequency and amplitude. Another coverslip is placed $1000 \mu \mathrm{m}$ in front of the actuated one. The back surface $\left(z_{0}\right)$ of the stationary coverslip provides the reference phase $\varphi\left(z_{0}, t\right)$, whilst $\varphi(z, t)$ is measured on the front surface $(z)$ of the actuated coverslip.

As seen in Fig. 1.B, a glass made microfluidic channel filled with the GNR solution (Nanopartz Inc., 1014 particles $/ \mathrm{mL}$, peak absorption wavelength $800 \mathrm{~nm}$ ) serves as the sample for PT-OCT investigation and imaging the photothermal trapping of GNRs in clear media (DI water). A 10x microscopy image of the aggregated GNRs in the glass channel is presented in Fig. 1.D. The aggregation resulted from the photothermal trapping of the Ti:Sa beam to the GNRs.

As seen in Fig. 1.C, a piece of pork tissue mounted on a coverslip serves as the sample for PT-OCT investigation and imaging the photothermal trapping of GNRs in the biological tissue sample. The GNR solution was injected into the tissue 5 minutes before the measurement. The experiment was performed in the common path OCT configuration. The front surface of the coverslip serves as the reference arm reflector, whilst the back surface of it provides the reference phase $\varphi\left(z_{0}, t\right) . \varphi(z, t)$ was detected at different axial locations in the tissue sample to locate GNRs.

\section{Results}

\subsection{Calibration}

To calibrate the phase sensitive OCT system, we designed three LabVIEW programmes to manipulate the acquired data in parallel. Each LabVIEW programme retrieved one of the $\Phi(z, t), \Delta \Phi(z, t)$ and $\left|\mathrm{p}\left(f_{0}\right)\right|$ quantities and produced their traces. We measured the amplitudes of these traces and took them into Eq. (3), Eq. (4) and Eq. (5) respectively to calculate the vibration amplitudes $A$. As discussed in the theory section, each equation can be used independent for the purpose of obtaining $A$. Here, the obtained experimental results of $A$ from all three LabVIEW programmes are plotted in the same graph and are compared with the data sheet of the piezo actuator, as shown in Fig. 2. 


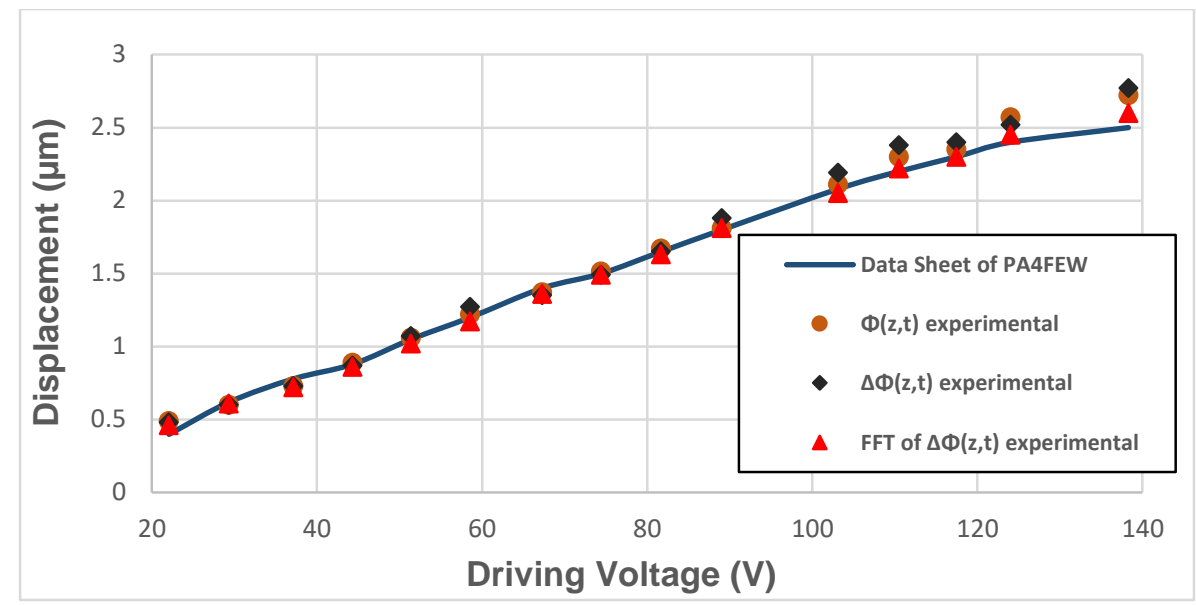

Fig. 2. System calibration. Measured amplitudes of traces of $\Phi(z, t), \Delta \Phi(z, t)$ and $\left|\mathrm{p}\left(f_{0}\right)\right|$ are introduced into Eq. (3), Eq. (4) and Eq. (5) respectively to calculate the vibration amplitudes $A$. Results (colored dots) obtained from three equations are compared with the data sheet (blue line) of the actuator.

To obtain the $\left|\mathrm{p}\left(f_{0}\right)\right|$ trace, FFT is performed on the last 1024 values of the $\Delta \Phi(z, t)$ trace. Sinusoidal signals with a fixed frequency of $5 \mathrm{~Hz}$ and selected voltages (from $20 \mathrm{~V}$ to $140 \mathrm{~V}$ ) were applied to the actuator. The actual displacements produced by the actuator cover a range of $0-3 \mu \mathrm{m}$. As seen in Fig. 2, from $20 \mathrm{~V}$ to $90 \mathrm{~V}$, the displacement values $A$ measured by three methods match the actual vibrating amplitude of the actuator. From $90 \mathrm{~V}$ upwards, compared with the other two methods, results obtained by the FFT method (triangular markers) exhibit better agreement with the blue trace (provided by the actuator data sheet). This is due to the inherently averaging nature of the FFT method since each FFT measurement is performed on $1024 \Delta \Phi(z, t)$ data obtained from 1024 measurements of $\Delta \Phi(z, t)$. In contrast, compared with the FFT method, the other two methods are more likely to be subject to the movement error of each single vibration of the actuator.

Because of its better accuracy performance, we chose the FFT method to measure the displacement sensitivity of the OCT system. To evaluate the measured displacement sensitivity, the measured value is then compared with the values characterised using the two mathematical models (described in section 5.1). Displacement sensitivity and its variation with the overall system operating speed, as well as defocus, are discussed in section 5.1 and section 5.2.

\subsection{Photothermal response of GNR}

As shown in Fig. 1, the measurements were made on two separate glass microfluidic channels, one filled with pure DI water and the other one filled with the GNR solution (Fig. 1.B). The air/glass interface of the microfluidic channel provided the reference phase $\varphi\left(z_{0}, t\right)$, while the signal phase $\varphi(z, t)$ was measured on the glass/solution interface. Though it has concluded from the experiments discussed above that the common path configuration exhibits a better displacement sensitivity than the separate reference arm configuration, the front glass of the microfluidic channel facing to the objective is too thick to be used in the common path configured system. Thus, measurements in this section were taken in the separate reference arm configuration, with both galvo-scanners disabled and an A-scan acquisition rate of $500 \mathrm{~Hz}$.

\subsubsection{Photothermal response of GNRs to Ti:Sa modulation frequency}

To investigate the photothermal response of the GNR solution to the modulation frequency of the Ti:Sa beam, we measured the differential phase $\Delta \Phi(z, t)$ variation over time with the Ti:Sa beam modulated by an optical chopper wheel (Fig. 1). When it rotates, the optical chopper wheel periodically interrupts the Ti:Sa beam travelling to the GNR sample. By doing this, it introduces a modulation to the photothermal response of the GNR sample to the Ti:Sa beam. Since the wheel is composed of metal spokes with sharp edges radiating from the hub, its rotation creates a square-wave modulation to the Ti:Sa illumination. Thus, an output signal with a square-wave appearance is obtained. 

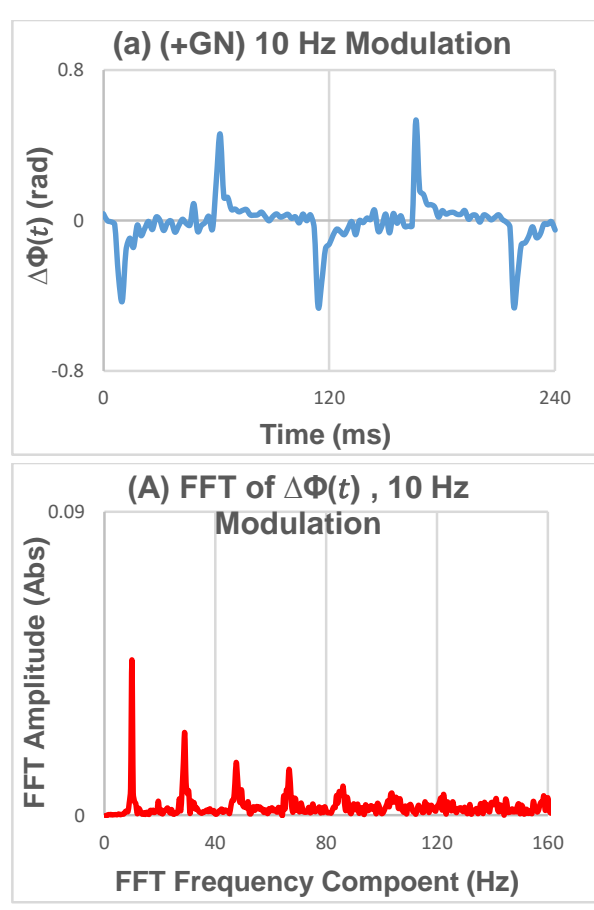

(b) (+GN) $20 \mathrm{~Hz}$ Modulation

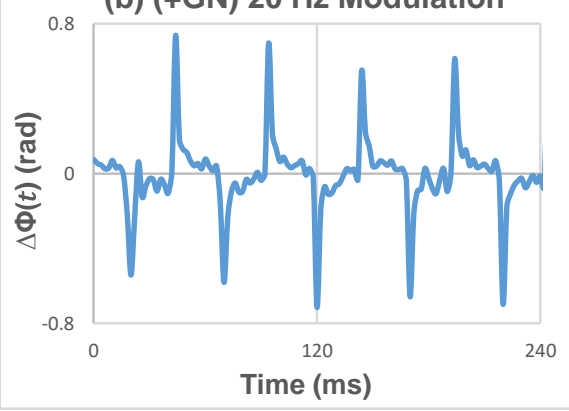

(B) FFT of $\Delta \Phi(t), 20 \mathrm{~Hz}$

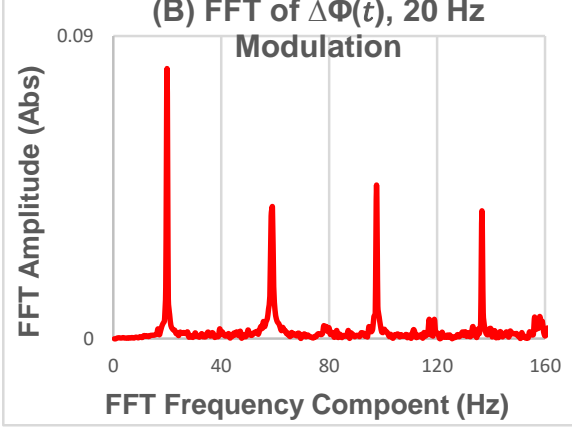

(c) (-GN, DI water) $20 \mathrm{~Hz}$

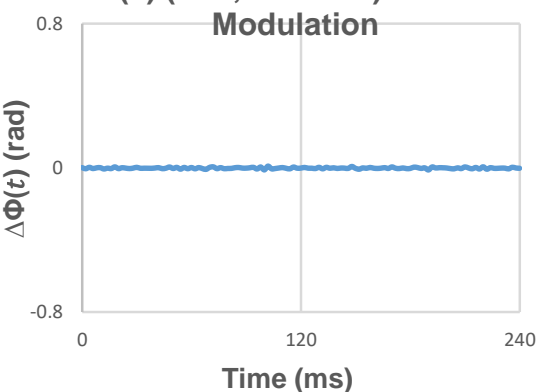

(C) FFT of $\Delta \Phi(t), 20 \mathrm{~Hz}$

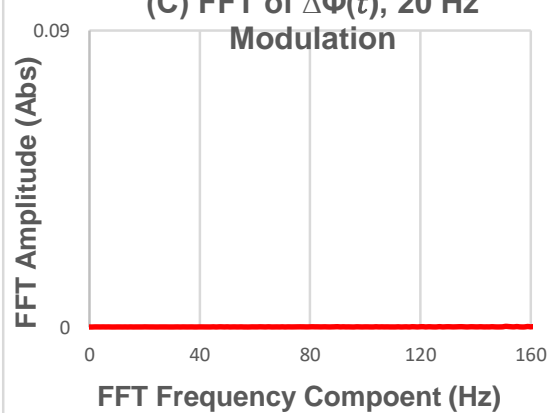

Fig. 3. Measured phase differential $\Delta \Phi(z, t)$ (a-c, in blue). FFT of $\Delta \Phi(z, t)$ (A-C, in red).

As mentioned above, since the trace of the output phase $\Phi(z, t)$ shows discontinuities due to the random noise term $\varphi$ in Eq. (2), the trace of the phase differential $\Delta \Phi(z, t)$ was used as the output signal. In this case, the expected shape of the output trace should be the differentiation of square-waves. The obtained results are presented in Fig. 3. Fig. 3(a) and Fig. 3(b) show $\Delta \Phi(z, t)$ traces measured at Ti:Sa modulation frequencies of $10 \mathrm{~Hz}$ and $20 \mathrm{~Hz}$. As seen, they present expected spike-like form which is in agreement with the differential of a square-wave. The positive spikes correspond to rising edges of square-waves, and the negative spikes correspond to falling edges of square-waves. The spike frequency is identical to that of the chopper wheel.

Fig. 3(c) shows the result of the channel filled with pure DI water, which appears generally featureless, indicating the lack of photothermal response when GNRs are absent. To enhance the SNR of measurements, FFT was performed on the $\Delta \Phi(z, t)$ trace. Fig. 3(A,B,C) show the frequency spectra of the corresponding $\Delta \Phi(z, t)$ traces for each condition in Fig. 3(a,b,c). As seen, the highest peak in each graph locates at the modulation frequency of the chopper wheel. According to the differentiation property of Fourier Transform, the FFT of the differentiation of a function has the same frequency components of the FFT of the original function but different amplitudes. Thus, in our case, the expected frequency components of the FFT of $\Delta \Phi(z, t)$ should be same as the frequency components of the FFT of square-waves. Since the FFT of square-waves is composed of only odd harmonics, the expected FFT of the $\Delta \Phi(z, t)$ trace should be composed of only odd harmonics. Limited by the A-scan operation rate $(500 \mathrm{~Hz})$ of the system, the highest detectable Ti:Sa modulation frequency was $250 \mathrm{~Hz}$. We fixed the Ti:Sa output power at $119.4 \mathrm{~mW}$ and varied the modulation frequency of the chopper wheel. The amplitude/height of the FFT peak at the modulation frequency is plotted against the Ti:Sa modulation frequency $(5-240 \mathrm{~Hz})$ in Fig. 4. 


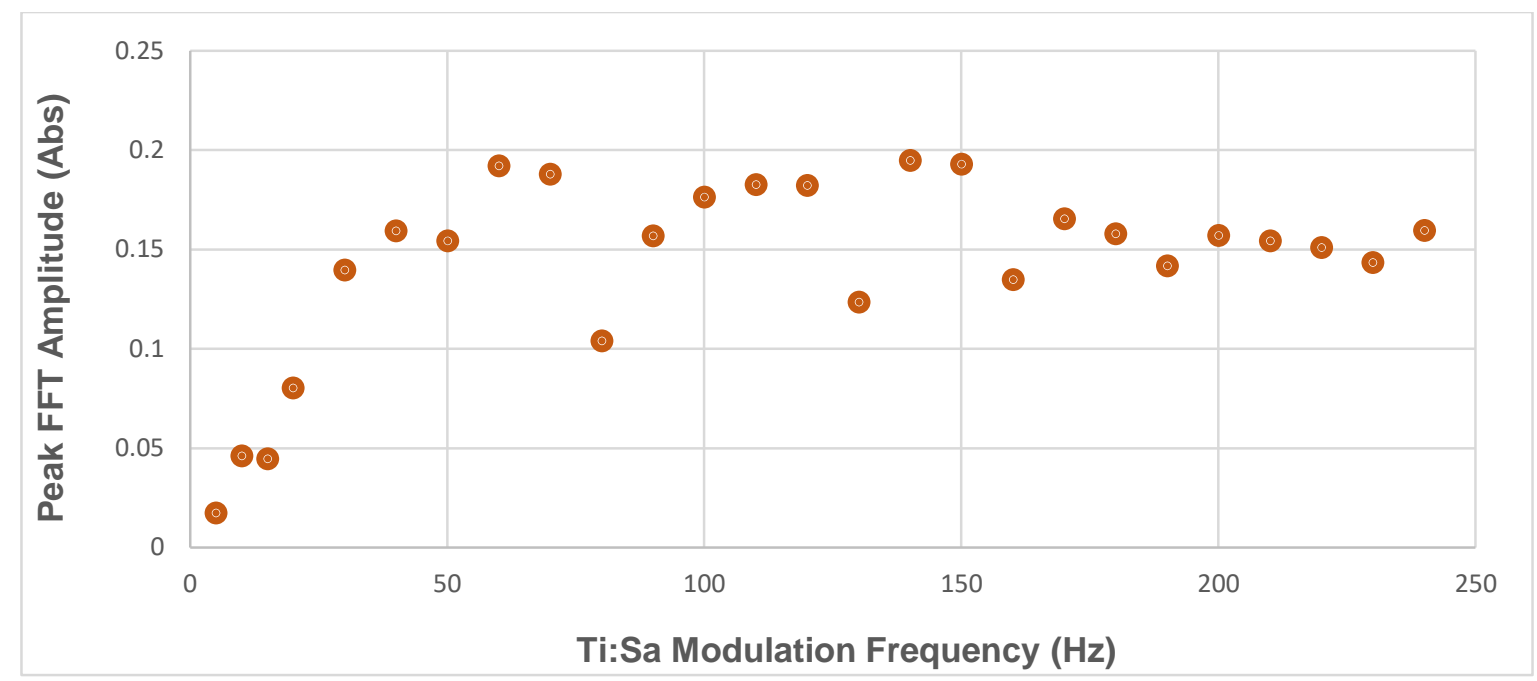

Fig. 4. Amplitude of the FFT peak at the Ti:Sa modulation frequency against the Ti:Sa modulation frequency.

As seen, the employed GNR solution exhibits (in broad terms) a superior photothermal response in the range 60-240 $\mathrm{Hz}$ compared to the range $5-50 \mathrm{~Hz}$. Though occasional dips exist on the trace in the range $60-240 \mathrm{~Hz}$, we have observed no evidence to link them in the response to a physical change (periodic or otherwise) in the measurement system.

\subsubsection{Photothermal response of GNRs to optical power of Ti:Sa beam}

We keep the Ti:Sa modulation frequency fixed at $220 \mathrm{~Hz}$ and increasingly attenuate the Ti:Sa power on the GNR sample using a neutral density (ND) filter (Fig. 1) in order to evaluate the minimum excitation power required to excite a detectable photothermal response of the GNR solution. Fig. 5 shows the FFT of $\Delta \Phi(z, t)$ for three different power values. As seen, the larger the attenuation, the smaller the SNR. Fig. 5(c) indicates that the minimum excitation power capable of inducing the photothermal response of GNRs is $0.583 \mathrm{~mW}$. This value is in line with the latest reported minimum Ti:Sa power $(0.5 \mathrm{~mW})$ capable of photothermal trapping GNRs [7].
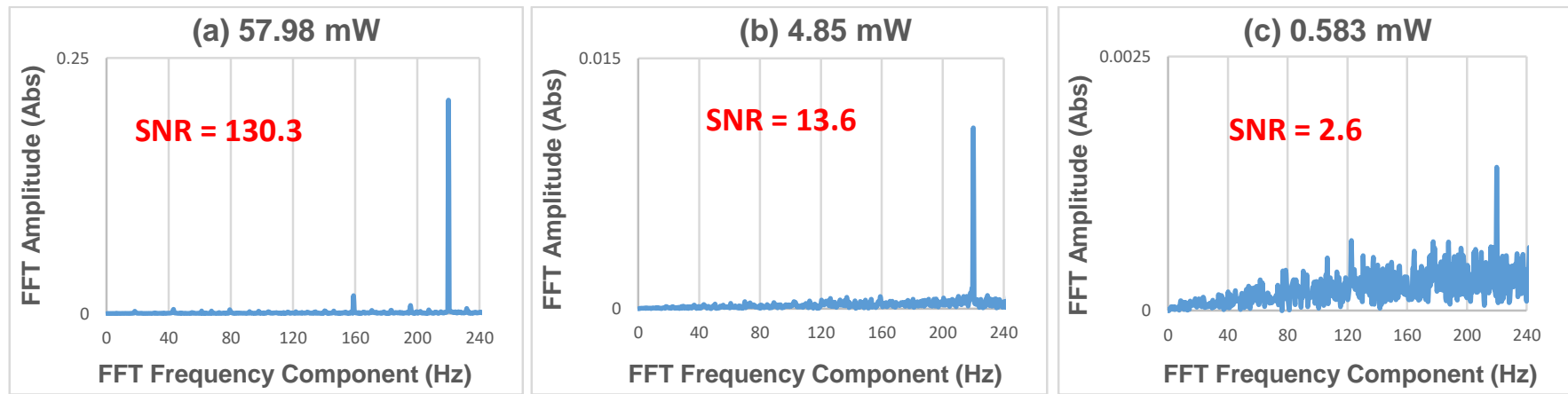

Fig. 5. FFT of $\Delta \Phi(z, t)$ for different Ti:Sa excitation power. Ti:Sa modulation frequency: $220 \mathrm{~Hz}$. 


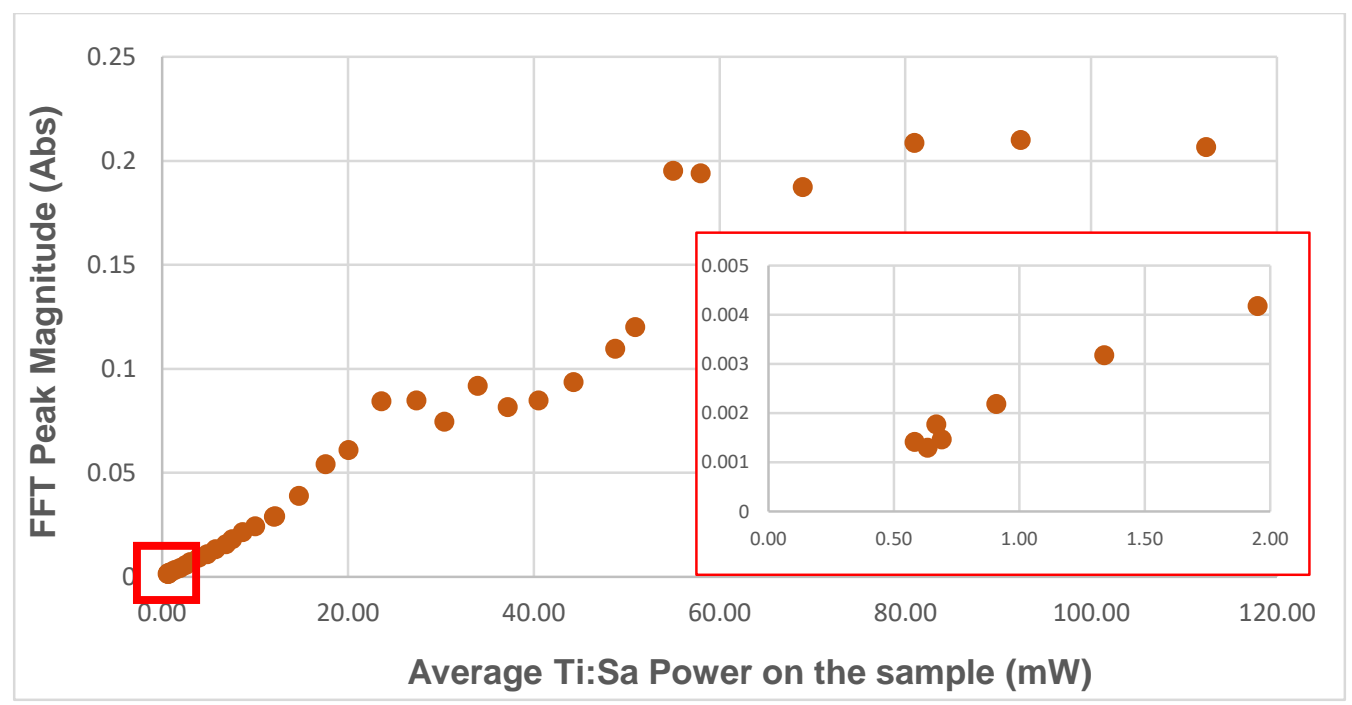

Fig. 6. Amplitudes of FFT peaks against average Ti:Sa power on the GNRs sample. Insert: zoom into the graph for power less than $2 \mathrm{~mW}$.

The amplitude of the FFT peak is plotted against excitation power in Fig. 6. As seen, below the power of $60 \mathrm{~mW}$, a decrease in the Ti:Sa excitation power results in a decrease of the signal. Above $60 \mathrm{~mW}$ power, some saturation of the photothermal response of GNRs takes place. As seen in the inset, below the power of $1 \mathrm{~mW}$, the Ti:Sa induced photothermal response of GNR is still detectable.

\subsection{PT-OCT for imaging photothermal trapped GNRs in clear media}

After the excitation beam was applied to the GNR solution, an aggregation of GNRs resulting from the photothermal trapping was observed (Fig. 1.D). The aggregation of GNRs is attributable to the self-assisted photothermal trapping of GNRs under the twophoton excitation. The TPL detection has been reported to image the trapping process [7]. As an alternative method to TPL, we demonstrate below that the PT-OCT system can be used to image aggregated GNRs resulted from the photothermal trapping. To this end, we performed imaging while the photothermal response of GNRs was either enabled or disabled by the shutter in Fig. 1. The OCT system was configured with a separate reference arm. As a 3D data volume containing the phase information of the sample was reconstructed, adequate data acquisition and operation speeds were required. In Section 5.1 we show that from the choice of settings available, a camera exposure time at $27.8 \mu$ s results in an adequate data acquisition speed for 3D imaging and an equivalent OCT displacement sensitivity of $7.83 \mathrm{~nm}$. The air/glass interface of the channel (Fig. 1.B) provides the reference phase $\varphi\left(z_{0}, t\right)$. As seen in Fig. 1.D, the combined OCT and Ti:Sa beams are scanned by two galvo-scanners over an area of $60 \times 60 \mu \mathrm{m}$ over the aggregated GNRs in the channel. The slow galvo-scanner (Fig. 1) determines a 3D frame rate of 0.5 frames/s. The fast galvo-scanner performs a line scan rate of 90 cycles/s $(11.1 \mathrm{~ms} /$ line $)$, equivalent with the beam integrating each pixel for $65 \mu \mathrm{s}$. A LabVIEW programme was created to assemble all collected A-scans into a 3D data set. An en-face image $(170 \times 170$ pixels, 0.35 $\mu \mathrm{m} /$ pixel) displaying phase matrix $-\pi \leq \Phi_{t}\left(x_{m}, y_{n}\right) \leq \pi$ is sliced at the glass/GNR boundary from the 3D data set, where $x_{m}, y_{n}$ are the lateral image coordinates. After a 3D frame interval of $\Delta t=2 \mathrm{~s}$, another phase matrix $\Phi_{t+\Delta t}\left(x_{m}, y_{n}\right)$ at the same boundary in a new 3D frame is obtained. The difference $\delta \Phi\left(x_{m}, y_{n}\right)$ between successive matrices is calculated as:

$$
\delta \Phi\left(x_{m}, y_{n}\right)=\Phi_{t+\Delta t}\left(x_{m}, y_{n}\right)-\Phi_{t}\left(x_{m}, y_{n}\right)
$$

where $-2 \pi \leq \delta \Phi\left(x_{m}, y_{n}\right) \leq 2 \pi$. Since the LabVIEW sub-VI being used to map the phase values into the brightness variation in the en-face image automatically scales negative $\delta \Phi\left(x_{m}, y_{n}\right)$ values into 0 , the information in the range of $-2 \pi \leq \delta \Phi\left(x_{m}, y_{n}\right) \leq 0$ is lost in the image. In this case, all features presented by negative values (between $-2 \pi$ and 0 ) of $\delta \Phi\left(x_{m}, y_{n}\right)$ would be scaled to 0 , showing as black with no distinguishable brightness variation in the produced images. In order to show the features with negative values of $\delta \Phi\left(x_{m}, y_{n}\right)$, a constant $\pi$ is then added to all $\delta \Phi\left(x_{m}, y_{n}\right)$ values before they are mapped to the grayscale to build en-face images in Fig. 7. In this case, $\delta \Phi\left(x_{m}, y_{n}\right)=-\pi$ is scaled to 0 , and $\delta \Phi\left(x_{m}, y_{n}\right)=3 \pi$ is scaled to 255 in the produced en-face images. Adding $\pi$, when $\delta \Phi\left(x_{m}, y_{n}\right)=-\pi, \delta \Phi\left(x_{m}, y_{n}\right)+\pi=0$ corresponds to an intermediate grayscale value in the image. 
When the shutter is closed, as shown in Fig. 7(A,a), GNRs present no photothermal response. In this case, the produced enface image displays no difference between successive acquisitions. While the Ti:Sa beam is blocked by the shutter, although GNRs do not give a photothermal response, phase differences are still created by laterally scanning the OCT beam over the borders between GNR areas and voids, and the borders are shown as thin contours in Fig. 7(A,a). When the shutter is switched from closed to open, the Ti:Sa beam triggers the photothermal response of GNRs, and the induced phase difference is imaged, as shown in Fig. 7(B,a). As seen, the bright area is covered by GNRs exhibiting a strong photothermal response to the Ti:Sa beam. Thus, the aggregated GNRs resulted from the photothermal trapping are imaged by the PT-OCT system. Keeping the shutter open, the next image in Fig. 7(C,a) is mostly grey. This is because the GNRs are in an equilibrium state generating photothermal responses. Therefore, no phase difference is induced between successive scanning frames of PT-OCT. When the shutter is switched from open to closed, the induced difference is displayed in Fig. 7(D,a). The dark region in Fig. 7(D,a) is bright in Fig. 7(B,a), and the bright area in Fig. 7(D,a) is dark in Fig. 7(B,a) due to an inverted order of the photothermal response.

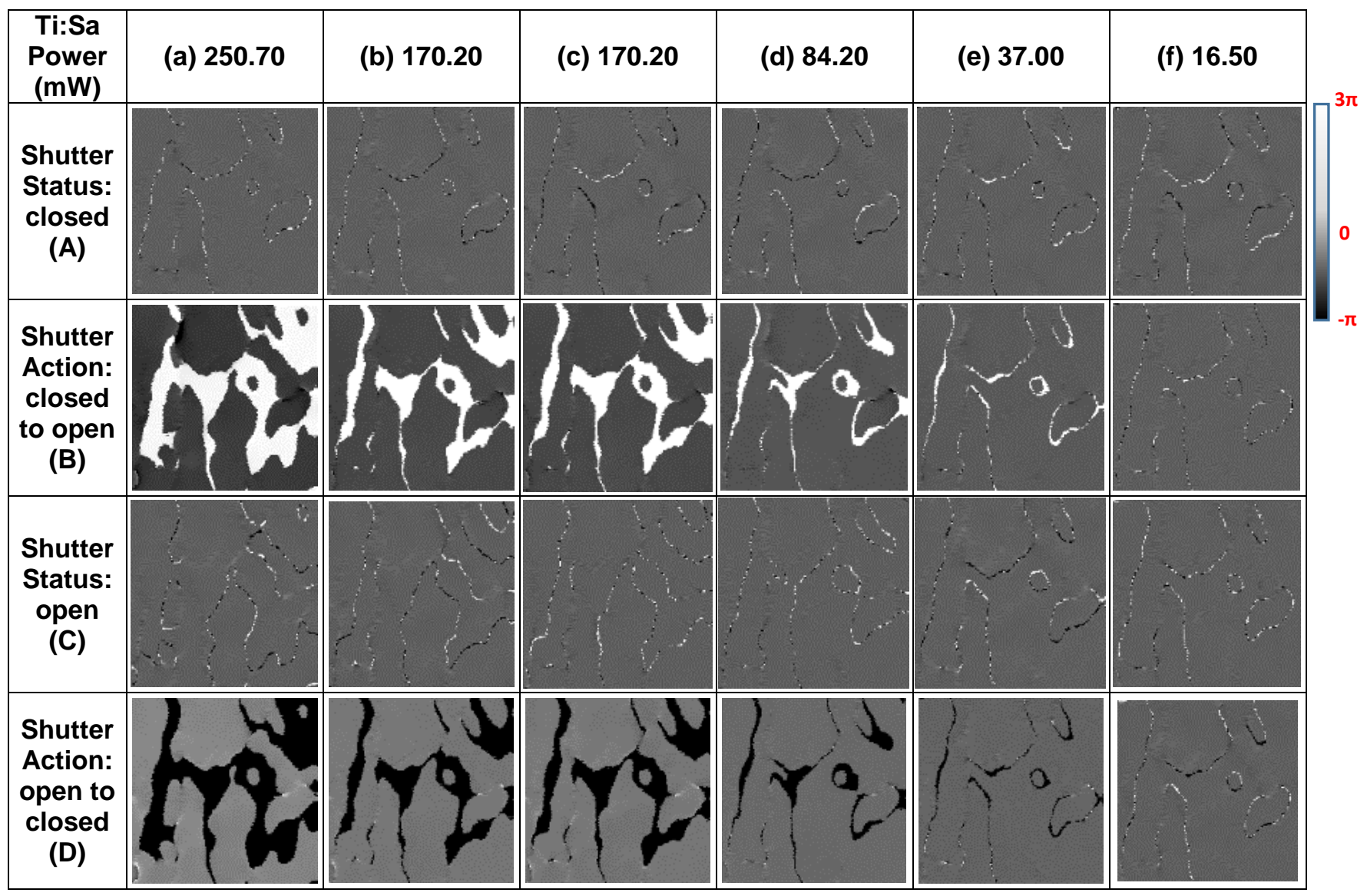

Fig. 7. En-face phase images of PT-OCT, displaying $\delta \Phi\left(x_{m}, y_{n}\right)+\pi$, at the glass/GNR boundary over the aggregated GNRs resulted from photothermal trapping. Image size: $170 \times 170$ pixel, $60 \times 60 \mu \mathrm{m}$.

Taking the shutter "from closed to open, then from open to closed" as a cycle, experiments were conducted in a series of such cycles with the power of the Ti:Sa beam progressively attenuated between cycles. As seen in Fig. 7 column (a-f), the smaller the excitation power, the smaller the areas of bright sectors. As seen in Fig. 7 column (f), when the excitation power is as low as 16.5 $\mathrm{mW}$, the difference between images in this cycle could be barely seen, indicating that the minimum power used in the PT-OCT system should be higher than this value. Images in Fig. 7 column (b) and Fig. 7 column (c) were acquired intentionally with the same power of $170.2 \mathrm{~mW}$. The similarity between corresponding images indicates that the measurements are highly repeatable. The drawback of the PT-OCT is that the absolute phase value $\Phi_{t}\left(x_{m}, y_{n}\right)$ is restricted between $-\pi$ and $\pi$. All phase values larger 
than $\pi$ are forced to $\pi$, and all phase values smaller than $-\pi$ are forced to $-\pi$. Thus, the PT-OCT is able to image the aggregation of GNRs but without offering any information on the thickness of the aggregated GNRs. The thickness of GNRs may offer information about the strength of photothermal trapping, in addition to the location of the aggregated GNRs in the en-face images (Fig. 7) which our method can show.

\subsection{PT-OCT for investigating photothermal trapping of GNRs in biological tissue}

Compared with freely diffusing GNRs in DI water, GNRs diffusing in biological tissue have more complex surroundings. To investigate the photothermal trapping of GNRs in biological tissue, we performed the PT-OCT measurement on a piece of pork tissue injected with the GNR solution. The sample preparation process is described in section 3.2 and Fig. 1.C. As shown, the front surface of the coverslip serves as the reference arm reflector for the common path configuration, and the back surface of it provides the reference phase $\varphi\left(z_{0}, t\right)$. Since the coverslip is thin, the system was set up in the common path configuration to benefit from a better displacement sensitivity (The choice of the configuration is discussed in section 5.1). To enhance the displacement sensitivity, the tightest beam focus is adjusted so that it is located inside the tissue (as discussed in section 5.2). Incident on a single lateral position on the sample, the Ti:Sa beam is modulated at $120 \mathrm{~Hz}$ by the chopper wheel (Fig. 1). To search for the depth at which GNRs are located, we varied the detecting depth $z$ (from the glass/tissue interface to the tissue/air interface) at which the $\Delta \Phi(z, t)$ signal is retrieved, while monitoring the FFT trace of $\Delta \Phi(z, t)$. When we reached the depth of $800 \mu \mathrm{m}$ below the glass/tissue interface, a peak at $120 \mathrm{~Hz}$ appeared in the FFT profile, indicating that GNRs exist at this depth. However, the height of the peak varied with time. This is shown by the FFT plots in Fig. 8, obtained every 5 seconds in a measurement sequence lasting 30 seconds.

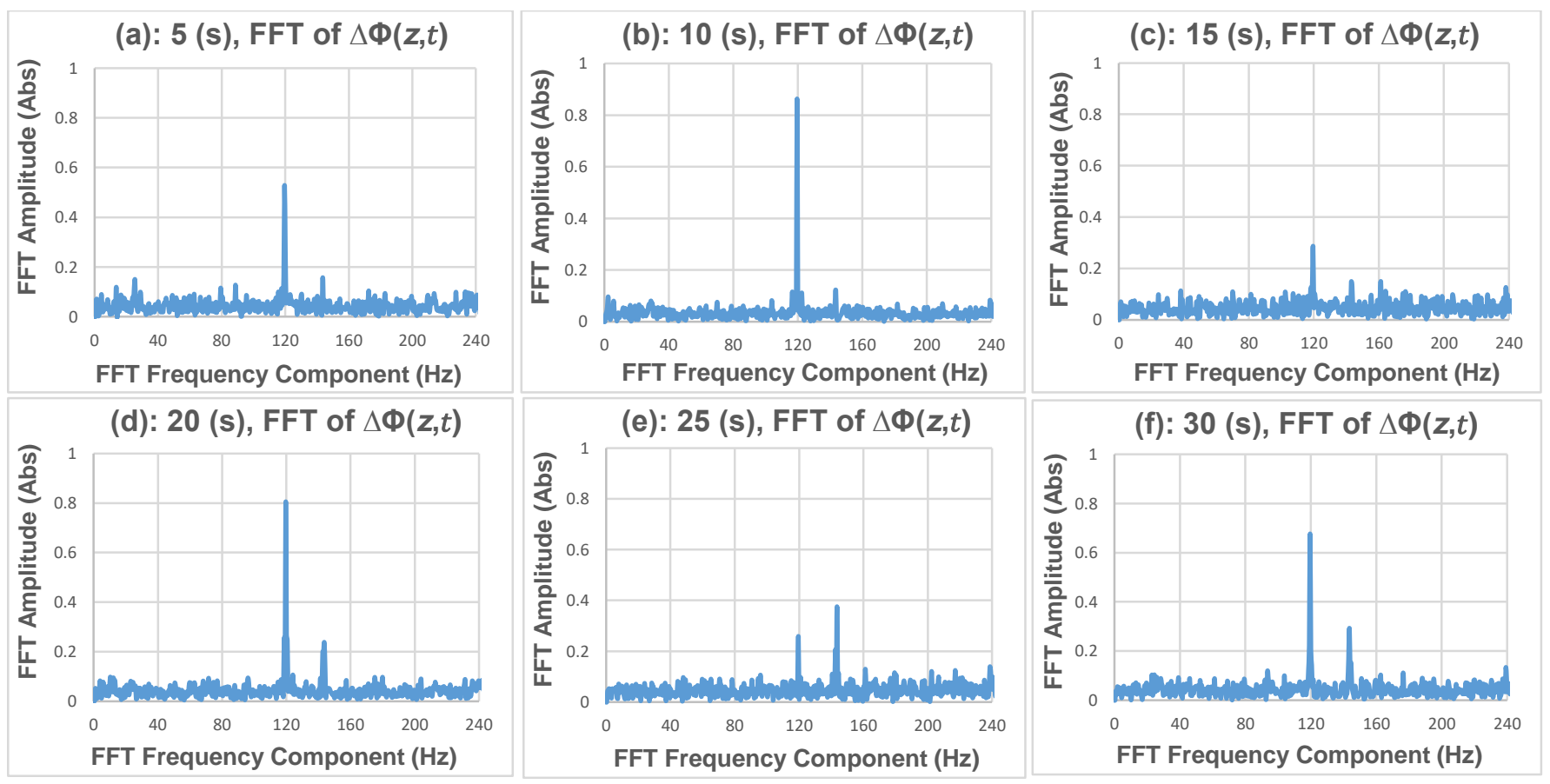

Fig. 8. FFT of $\Delta \Phi(z, t)$ at the depth of $800 \mu \mathrm{m}$ in the tissue injected with GNRs. The Ti:Sa beam was modulated at $120 \mathrm{~Hz}$. Graphs were obtained every 5 seconds in a measurement lasting 30 second.

As seen in Fig. 8, the amplitude of the FFT peak fluctuates during the 30 seconds measurement. Unlike the stable FFT peak obtained in section 4.1, where GNRs were diffusing in DI water, the fluctuation in Fig. 8 suggests that the photothermal trapping induced movement of GNRs in the biological sample is affected by the time evolution of the tissue structure. Thus, the concepts used to explain the photothermal trapping of GNRs in clear media in the reference [7] may not fully explain the photothermal trapping of GNRs in biological tissue. In Fig. 8, for instance, a broad overall reduction in amplitude such as (b) to (c) can be 
ascribed to changes in the light transmission properties of the tissue close to the focal spot (tissue becomes more opaque as it is heated due to denaturation of protein structure). In the meanwhile, the increases in amplitude such as (c) to (d) and subsequently (e) to (f) suggest a degree of complexity in the photothermal trapping induced movement of GNRs in tissue which is consistent with changes in the shape of localised GNR accumulations but should not necessarily be interpreted as a manifestation of a convective flow similar to that described in clear media in [7].

The method and operating parameters used in section 4.2 were employed to carry out the PT-OCT imaging of photothermal trapped GNRs in the pork tissue. In Fig. 9, row A and row B show grayscale images, representing the $\delta \Phi\left(x_{m}, y_{n}\right)$ array, that were obtained inside the tissue sample from two different layers. The depth corresponding to row A is $189 \mu \mathrm{m}$ inside the tissue and the depth corresponding to row $\mathrm{B}$ is $431 \mu \mathrm{m}$ inside the tissue.

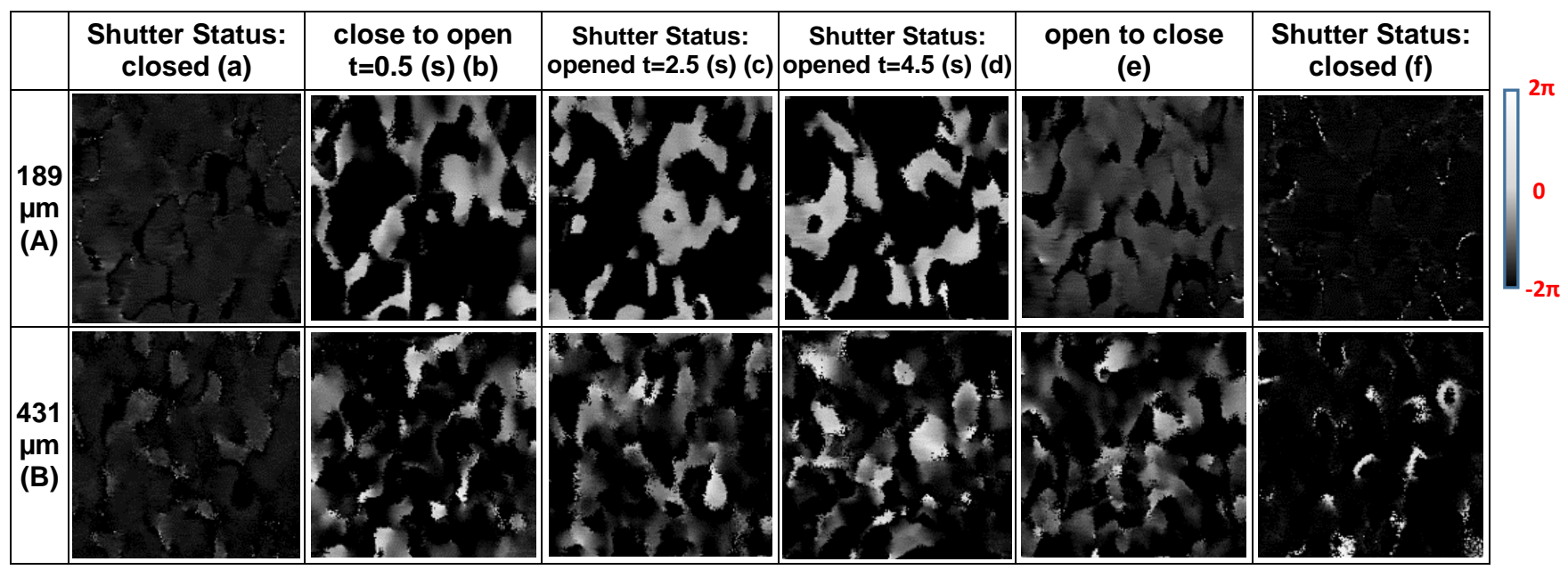

Fig. 9. En-face PT-OCT images displaying $\delta \Phi\left(x_{m}, y_{n}\right)=\Phi_{t+\Delta t}\left(x_{m}, y_{n}\right)-\Phi_{t}\left(x_{m}, y_{n}\right)$. Images display the depths of $189 \mu \mathrm{m}$ and $431 \mu \mathrm{m}$ in the tissue. Columns (b-d) were obtained 0.5 second, 2.5 seconds and 4.5 seconds after the shutter was opened. Image size: $60 \mu \mathrm{m} \times 60$ $\mu \mathrm{m}$.

Images in Fig. 9 column (a) are mostly dark when the shutter is closed. The patterns of bright shades in Fig. 9 column (b), analogous to Fig. 7 row (B), show the location of GNRs when the shutter is switched from closed to open. The PT-OCT was used for imaging GNRs at different depths in the biological tissue. Images in Fig. 9 columns (b-d) are obtained at different time after the shutter was opened. Unlike the images in Fig. 7 row (C) that present no pattern, Fig. 9 columns (b-d) contain varied patterns of bright shades. The effect of varied patterns is expected, which also agrees with the fluctuating peaks in Fig. 8 . These results presented in Fig. 8 and Fig. 9, conducted with GNRs in the tissue sample, can be explained based on two reasons. Firstly, the fluctuating peaks in Fig. 8 and the varied patterns in Fig. 9 indicate that the photothermal trapping has induced movements of GNRs in the tissue, movement that is somehow affected by the tissue morphology. This effect is not observed with the GNRs in the DI water, where the signal peak is stable in time. Secondly, those effects can be ascribed to the changes in the light transmission properties of the tissue close to the focal spot where the tissue is being cooked during the excitation process so that the heat induced denaturation of the protein structure increases the complexity in the photothermal trapping of GNRs. The net result is the fluctuating peaks in Fig. 8 generated over time while the Ti:Sa beam was modulated at $120 \mathrm{~Hz}$, and the varied patterns in Fig. 9 columns (bd) generated over time while the shutter was left open. When the shutter is switched from open to closed, the images in Fig. 9 column (e) should display a similar effect as that noticed in Fig. 7 row (D). However, due to the two reasons mentioned just above, the images obtained are not exact reversed replicas of any images recorded before. As seen in Fig. 9 column (f), when the shutter is left closed, images from both layers are mostly dark showing no feature, analogous to what happens in Fig. 7 row (C).

\section{Discussion}

Given that the photothermal effect of GNRs induced OPD variation can be of the order of a few nm only, it is important to choose the parameters of the PT-OCT system in a way that enhances the displacement sensitivity. Two factors playing an important role 
are the A-scan acquisition speed (linked to camera exposure time in our SD-OCT system), and the ability to cope with defocus at the GNR z-location, as discussed below.

\subsection{Displacement sensitivity - variation with A-scan rate for common path and separate reference arm OCT}

The smallest distinguishable phase fluctuation about an average DC value is a measure of the phase sensitivity $\sigma_{\phi}$ of a phase sensitive OCT system [24,38], which then determines the axial displacement sensitivity $\sigma_{z}$ according to Eq. (2) and Eq. (9). In this study, we employed two commonly used methods to characterise and estimate the phase sensitivity of the system.

The first method characterising the phase sensitivity comes from the perspective of the system noise level, where the fundamental limitation on the phase sensitivity arises from the signal-to-noise ratio (SNR) of the measurement procedure $[35,36]$. Under the assumption that the SNR of the measured OCT signal intensity satisfies SNR > 1, the phase variance $\sigma_{\phi}$ is given by $\sigma_{\phi}=\frac{1}{\sqrt{S N R}}$, and the path variance $\sigma_{z}$ can be expressed as [26,36,37]:

$$
\sigma_{z}=\frac{\lambda_{0} \sigma_{\phi}}{4 \pi n}=\frac{\lambda_{0}}{4 \pi n \sqrt{S N R}}
$$

where $\lambda_{0}=1050 \mathrm{~nm}$ is the central wavelength of the OCT light source and $n=1$ is the refractive index of air. In this study, based on the perspective of the system noise level, we use $\sigma_{z}$ to characterise displacement sensitivity of the system. To work out $\sigma_{z}$, the SNR in Eq. (9) was measured as the ratio of the A-scan peak of the stationary interface (Fig. 1.A) to its noise floor in the A-scan profile. It is widely accepted that an interferometer configured with a separate reference arm exhibits worse system noise floor than a common path configured system [26,36,37]. In addition, we found that the camera pixel sensitivity is another factor affecting the phase sensitivity of a SD-OCT system. Specifically, improved camera pixel sensitivity can be obtained by extending the camera exposure time, which in return reduces the data acquisition speed (A-scan rate). Consequently, it is important for us to find the optimum trade-off between the camera sensitivity and the operating speed of the system. Such a trade-off will offer an acceptable measurement sensitivity, and at the same time the system maintains its operating speed (A-scan rate) in hundreds of Hz. The Goodrich SUI camera in the system provides different pre-set operating modes, each of which has fixed settings of camera exposure time and pixel sensitivity, optimised in pairs. Thus, we measured the SNR in two interferometer configurations separately, and in each configuration we varied the camera exposure time settings. To implement the common path configuration, the reference arm (Fig. 1.A) was blocked, and the front surface of the stationary coverslip serves as the reference reflector. The measured SNR was taken into Eq. (9) to calculate $\sigma_{z}$. Results are compared in (b) and (c) in Fig. 10.

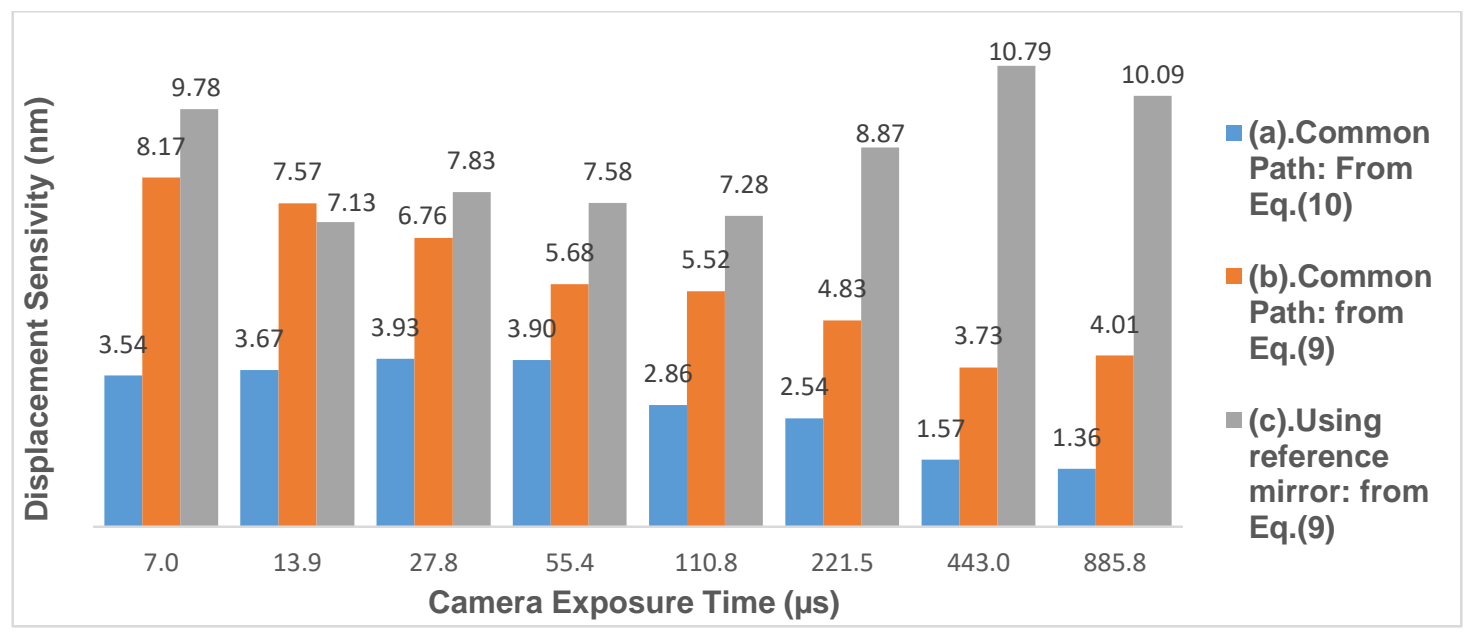

Fig. 10. Characterized displacement sensitivity. (a): Eq. (10), common path configuration. (b): Eq. (9), common path configuration. (c): Eq. (9), reference arm configuration.

The second method characterising the phase sensitivity comes from the perspective of the statistics of the output phase stability measured on a stationary interface. It has been reported that the phase fluctuation contributed by interferometric instabilities 
increases the phase noise floor and degrades the phase sensitivity [24,38]. Based on this perspective, the displacement sensitivity $\sigma_{z}^{\prime}$ can be expressed as $[24,38]$ :

$$
\sigma_{z}^{\prime}=\frac{\lambda_{0} \varphi_{S D}}{4 \pi n}
$$

where $\varphi_{S D}$ is the standard deviation of a set of successive phase values.

To work out $\sigma_{z}^{\prime}$ in Eq. (10), we performed A-scans $(500 \mathrm{~Hz})$ continuously at a fixed lateral location on the stationary glass surface (located at $z$ in Fig. 1.A), and then calculated the standard deviation $\varphi_{S D}$ of 1024 output phase values. All measurements were performed in the common path configuration with varied camera exposure time settings. Results are presented in histograms (a) in Fig. 10.

As seen in Fig. 10, the displacement sensitivity of the common path configured system (shown as (b) in Fig. 10) is better than that of the reference arm configured system (shown as (c) in Fig. 10). In the common path configured system (a) and (b), camera exposure time settings of $443 \mu \mathrm{s}$ and $886 \mu$ s provide the best displacement sensitivities. The displacements obtained from these two camera settings are similar and comparable in each set-up configuration, $1.57 \mathrm{~nm}$ to $1.36 \mathrm{~nm}$ for (a), $3.73 \mathrm{~nm}$ to $4.01 \mathrm{~nm}$ for (b), and $10.79 \mathrm{~nm}$ to $10.09 \mathrm{~nm}$ for (c). Practically, if the displacement sensitivity is comparable, a faster operating rate is always preferred. Thus, we used the setting of $443 \mu \mathrm{s}$ rather than the setting of $886 \mu \mathrm{s}$ for the photothermal measurements (Fig. 3, Fig. 4, Fig. 5, Fig. 6, Fig. 8) that do not involve galvo scanning. Furthermore, as discussed in section 5.3, as long as the geometry of the studied sample allowed, we chose the common path configuration to perform our measurements (Fig. 8 and Fig. 9) since histograms of (a) and (b) are always lower than that of (c) in Fig. 10.

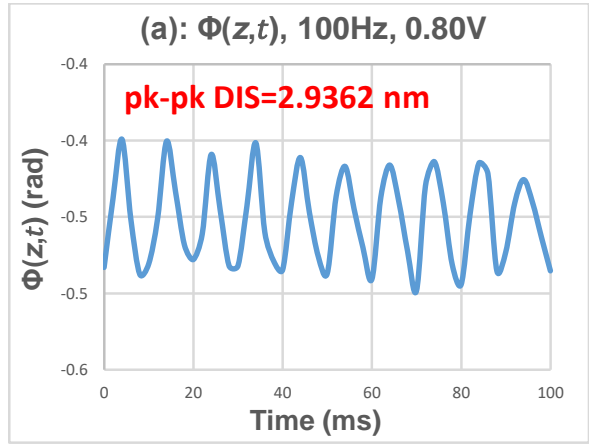

(d): FFT of $\Delta \Phi(z, t), 0.20 \mathrm{~V}$

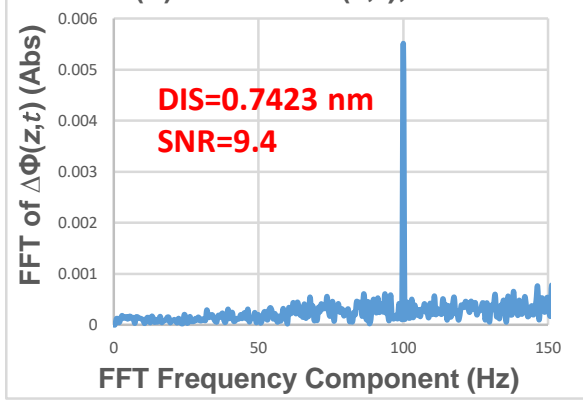

(b): $\Phi(z, t), 100 \mathrm{~Hz}, 0.40 \mathrm{~V}$

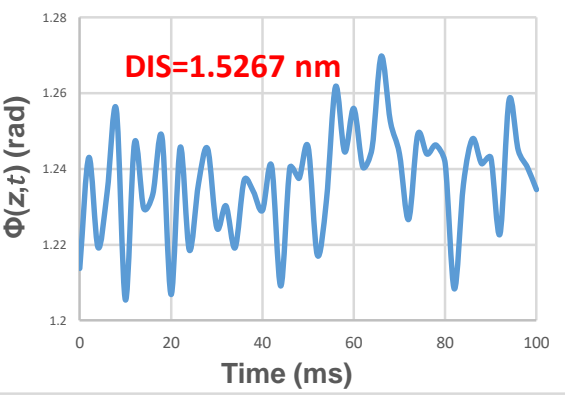

(e): FFT of $\Delta \Phi(z, t), 0.10 \mathrm{~V}$

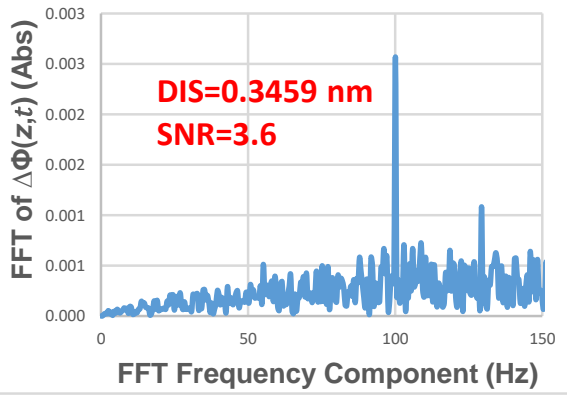

(c): FFT of $\Delta \Phi(z, t), 0.40 \mathrm{~V}$

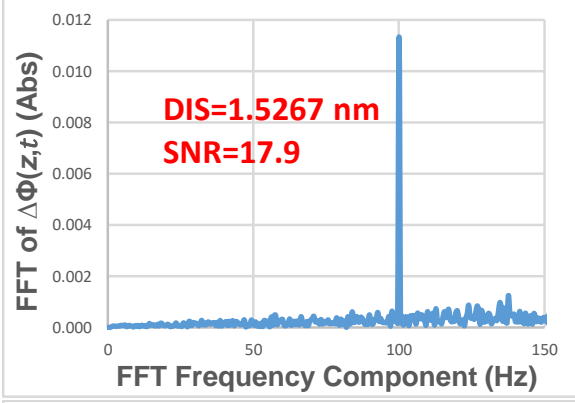

(f): FFT of $\Delta \Phi(z, t), 0.04 \mathrm{~V}$

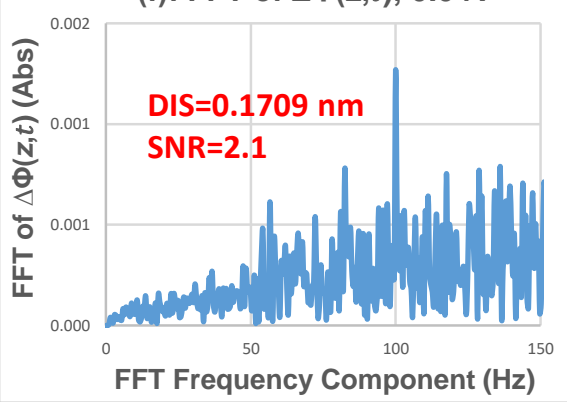

Fig. 11. DIS: displacement amplitude. (a): $\Phi(z, t)$ output trace at $0.8 \mathrm{~V}$. (b): $\Phi(z, t)$ output trace at $0.4 \mathrm{~V}$. (c-f): FFT of $\Delta \Phi(z, t)$ at driving voltages of $0.4 \mathrm{~V}, 0.2 \mathrm{~V}, 0.1 \mathrm{~V}$ and $0.04 \mathrm{~V}$ respectively. DIS in (a) is calculated using Eq. (3), and DIS in (b-f) is calculated using Eq. (5).

The sensitivity values plotted as (a) and (b) histograms in Fig. 10 are obtained in the same system configuration (common path configuration) but are calculated using Eq. (9) and (10) respectively, corresponding to the two different perspectives in characterising the phase sensitivity outlined above. To experimentally evaluate and compare the accuracies of these two perspectives, we performed measurements on the vibrating actuator (Fig. 1.A) driven by sinusoidal voltages at $100 \mathrm{~Hz}$ with selected voltages $(0.8 \mathrm{~V}, 0.4 \mathrm{~V}, 0.2 \mathrm{~V}, 0.1 \mathrm{~V}, 0.04 \mathrm{~V})$. For the voltages of $0.8 \mathrm{~V}$ and $0.4 \mathrm{~V}$, the LabVIEW programme outputting $\Phi(z, t)$ were used, and the produced traces of $\Phi(z, t)$ are presented in (a) and (b) in Fig. 11. As seen in Fig. 11(b), when the driving voltage 
is smaller than $0.4 \mathrm{~V}$, the $\Phi(z, t)$ trace losses the sinusoidal pattern. In this case, the method of outputting $\Phi(z, t)$ meets its sensitivity limitation. Then, for the voltages of $0.4 \mathrm{~V}, 0.2 \mathrm{~V}, 0.1 \mathrm{~V}, 0.04 \mathrm{~V}$, the LabVIEW programme outputting the FFT of $\Delta \Phi(z, t)$ were used, and the produced traces of FFT are presented in (c) - (f) in Fig. 11. All measurements were performed in the common path configuration with the camera set at $443 \mu \mathrm{s}$. The aim of the experiment is to search the smallest detectable displacement which will be then compared with the histograms (a) and (b) in Fig. 10.

Since the $\Phi(z, t)$ trace was not able to detect the sinusoidal vibration when the actuator was driven by the voltage smaller than $0.4 \mathrm{~V}$, instead of outputting the $\Phi(z, t)$ trace, we applied FFT on the $\Delta \Phi(z, t)$ trace to improve the displacement sensitivity further. As shown in each individual graph in (c) - (f) in Fig. 11, we measured the amplitude $\left|\mathrm{p}\left(f_{0}\right)\right|$ of the FFT peak that sits at the driving frequency of $100 \mathrm{~Hz}$, and took the obtained value into Eq. (5) to calculate the displacement amplitude $A$. As seen in Fig. 11(f), the detectable displacement using the FFT method can be as small as $0.17 \mathrm{~nm}$ with a SNR of 2.1. This displacement was produced when the actuator was driven at $0.04 \mathrm{~V}$.

\subsection{Displacement sensitivity - variation with defocus}

The phase sensitivity degradation with defocus plays an important role in phase microscopy [39]. In our common path configured system, the degradation was evaluated by measuring the displacement sensitivity while moving the detected boundary (located at $z$ in Fig. 1.A) axially away in steps of $0.05 \mathrm{~mm}$ from the reference interface (located at $z_{0}$ in Fig. 1.A). Eq. (10) was used to calculate the displacement sensitivity at each axial location. Results are presented in Fig. 12.

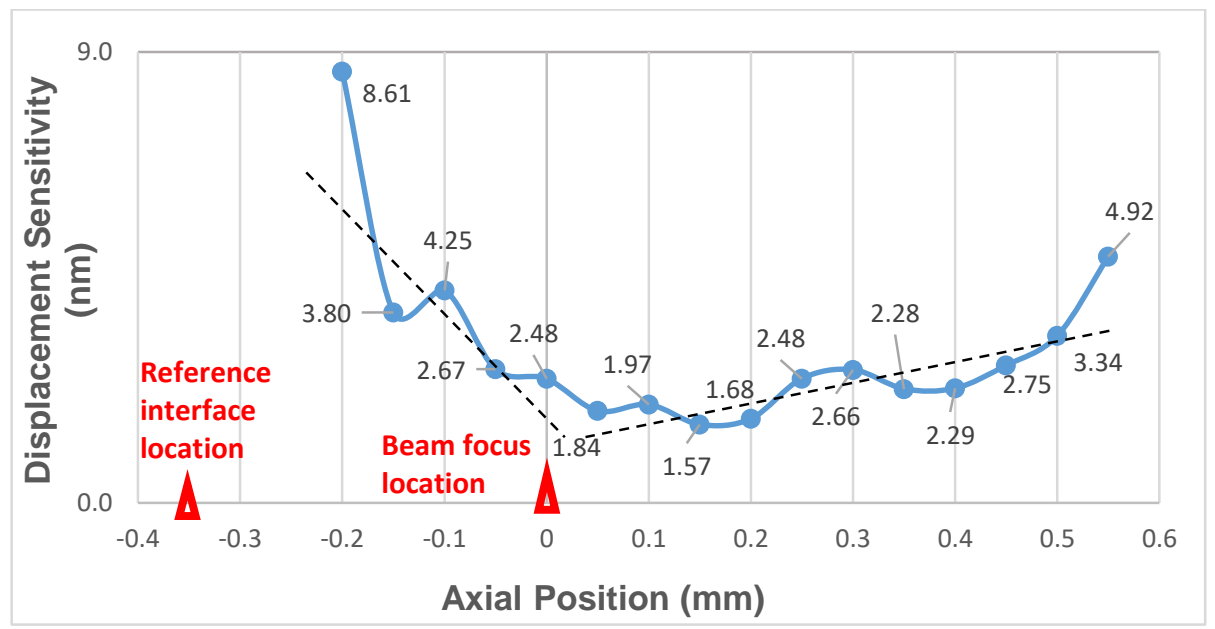

Fig. 12. Displacement sensitivity degradation with defocus. The reference interface $\left(z_{0}\right.$ in Fig. 1.A) is located at $-0.35 \mathrm{~mm}$ (left red triangle in the figure). The beam focus position is located at $0 \mathrm{~mm}$ (right red triangle in the figure). The displacement sensitivity is measured on the detected interface ( $z$ in Fig. 1.A) while it is placed at selected axial positions.

The "Axial Position" in Fig. 12 serves as the position coordinate in the axial direction. The stationary reference interface $\left(z_{0}\right.$ in Fig. 1.A) and the tightest beam focus are fixed at $-0.35 \mathrm{~mm}$ and $0 \mathrm{~mm}$ respectively. The displacement sensitivities were measured on the moving detected interface ( $z$ in Fig. 1.A) at selected axial locations. As seen, when the detected interface is placed $0.15 \mathrm{~mm}$ to the right of the beam focus, the best displacement sensitivity of $1.57 \mathrm{~nm}$ is obtained. Given by the fitting lines (black-dashed), the region to the right of the beam focus has a displacement sensitivity degradation of $1.72 \mathrm{~nm} / \mathrm{mm}$, whereas the region to the left of it has a value of $30.65 \mathrm{~nm} / \mathrm{mm}$. The degradation is clearly a consequence of the properties of the focusing optics being used. Based on the curve in Fig. 12, in the experiments (Fig. 8 and Fig. 9), the GNR sample is placed within the axial range $(-0.05 \mathrm{~mm}$ to $0.45 \mathrm{~mm}$ ) to make the experiment benefit from a sufficiently good displacement sensitivity performance.

\subsection{Choice of OCT configuration}

In our practice, the measurement parameters are significantly influenced by other factors, and the geometry of the sample is one the factors. Specifically, as proved in section 5.1, the common path configuration is always preferred because it exhibits a better 
displacement sensitivity $(1.57 \mathrm{~nm}$, camera: $443 \mu \mathrm{s})$ than the separate reference arm configuration (10.79 nm, camera: $443 \mu \mathrm{s})$. However, for PT-OCT imaging of GNRs in DI water (Fig. 3, Fig. 4, Fig. 5, Fig. 6, Fig. 7), we chose the separate reference arm configuration. That is because the employed microfluidic channel had a thickness of $400 \mu \mathrm{m}$, but the maximum imaging depth of the OCT system was only $1400 \mu \mathrm{m}$ in air. The geometry of the microfluidic channel does not fit the common path configuration setup as it does not allow placing the detected interface in the beam waist with the best displacement sensitivity (as discussed in section 5.2). Despite a poorer displacement sensitivity, the minimum average Ti:Sa power $(0.583 \mathrm{~mW})$ in PT-OCT capable of generating a detectable photothermal response of GNRs is still in line with the reported minimum Ti:Sa power $(0.5 \mathrm{~mW})$ for photothermal trapping of GNRs in [7] where the TPL detection is employed. Consequently, to investigate the photothermal trapping of GNRs, the PT-OCT should be superior to the TPL detection when common path configuration is employed.

In section 4.4, as the coverslip in contact with the tissue sample was only $100 \mu \mathrm{m}$ thick, the common path configuration was chosen. A longer camera exposure time may have improved the displacement sensitivity of the system but this would have also slowed down the A-scan acquisition speed. We found that the camera exposure time of $443 \mu$ s and the A-scan rate of $500 \mathrm{~Hz}$ resulted in an acceptable trade-off for obtaining an overall system performance. The depth of $800 \mu \mathrm{m}$, where GNRs were located by our PT-OCT method, is close to the maximum imaging depth of the system (1000 $\mu \mathrm{m}$ in tissue). Thus, the PT-OCT technology could be able to locate GNRs deep in the tissue if a higher resolution spectrometer was employed.

For PT-OCT imaging of the photothermal trapping of GNRs in DI water (section 4.3), the separate reference arm configuration was employed to accommodate the geometry of the microfluidic channel. A camera exposure time of $27.8 \mu \mathrm{s}$ was used, which could perform a maximum B-scan line rate of 200 lines/s, to satisfy our B-scan line rate of 90 lines/s. The system configuration and the camera exposure time result in a displacement sensitivity of $7.83 \mathrm{~nm}$ according to Fig. 10 (c). Compared with the best displacement sensitivity of $1.57 \mathrm{~nm}$, this degraded displacement sensitivity resulted in fewer measured phase values that fall between $-\pi$ and $\pi$. As discussed in section 4.3 , all phase values were wrapped between $-\pi$ and $\pi$. As a consequence, the phase wrapping had an effect on rescaling partial phase values used to build the en-face images in Fig. 7. Thus, the axial thickness of the aggregated GNRs, which is also linked to the strength of photothermal trapping, could be quantified by the PT-OCT technology but only by using a suitable algorithm for phase unwrapping, which is able to extend the depth range of obtained phase values. Thus, to improve the method for investigation and imaging the photothermal trapping of GNRs by PT-OCT, an effective phase unwrapping algorithm suitable for en-face imaging should be developed in future studies to remove the $2 \pi$ ambiguity. In addition, in the future study, with the help of the phase unwrapping algorithm, the PT-OCT could be employed to visualize the photothermal trapping process of GNRs.

By contrast, the common path configuration was chosen for imaging the photothermal trapping of GNRs in the tissue sample in section 4.4. This configuration has an improved displacement sensitivity of $3.93 \mathrm{~nm}$, resulting in more phase values in the range $[-\pi, \pi]$ that are used to build the images in Fig. 9.

In Fig. 8, the fluctuation of the FFT peak indicates that the photothermal trapping of GNRs is affected by internal variations of the tissue structure. This indication agrees with the varying pattern in Fig. 9 columns (b-d). Given by the varying pattern in Fig. 9 columns (b-d), when the shutter is held open, the photothermal trapping induced movements of GNRs in the tissue sample is affected by the permanent change of the tissue structure as well.

\section{Conclusion}

In this paper, we demonstrate that a spectrometer based PT-OCT system with sub-nanometre displacement sensitivity performance is capable of imaging the photothermal trapping induced aggregation of GNRs. We also evaluate the fundamental and hardware limits on the system displacement sensitivity as functions of SNR and output phase stability. By optimising the camera exposure time, the OCT system in a common path configuration is found to allow detection of axial displacements as small as $1.57 \mathrm{~nm}$. When a periodic movement is detected, and the Fourier analysis is carried out on the output phase, notable improvement is obtained in the displacement sensitivity of better than $0.17 \mathrm{~nm}$ (with SNR of 2.1). The system in a separate reference arm configuration is found to exhibit worse displacement sensitivity performance than the common path configuration. This is expected because a separate reference arm makes the output phase susceptible to mechanical or thermal fluctuations in both arms. Nevertheless, a separate reference arm configured system is still capable to image the pattern of aggregated GNRs in vitro. Using a separate reference arm configured PT-OCT system, it is found that the minimum Ti:Sa power able to induce the detectable photothermal response of the GNR solution is $0.5 \mathrm{~mW}$. This value is in good agreement with the reported minimum Ti:Sa power able to induce photothermal trapping of GNRs [7]. Thus, a common path configured PT-OCT system with better sensitivity performance should exceed the TPL detection in searching for the minimum Ti:Sa power in generating a detectable photothermal response of GNRs. 
We obtained en-face phase images as grayscale $\mathrm{x}-\mathrm{y}$ maps, displaying photothermally induced changes in GNRs and surrounding media. When comparing the phases before and after the Ti:Sa excitation, the locations of aggregated GNRs are represented as brighter shades, whereas locations with fewer or no GNRs present are represented as darker shades. To compare the image made by the PT-OCT with that made by the TPL, the lateral scanning area of the PT-OCT is set to be $60 \mu \mathrm{m} \times 60 \mu \mathrm{m}$ which is in line with that of the TPL $(66 \mu \mathrm{m} \times 66 \mu \mathrm{m}$ FOV $)$ [7]. Since the objective employed in the PT-OCT has the magnification of $10 \times$, the achieved lateral resolution in the image is not comparable to that of the TPL, in which $40 \times$ and $60 \times$ water objectives were employed [7]. Nevertheless, the image contrast of the PT-OCT image is better than that of the TPL image. This advantage of PTOCT is a consequence of the grayscale encoding of the difference between successive en-face phase maps, in which the background information is removed from the image by the subtraction, leaving only the short burst occurring during the Ti:Sa modulation process in the image. In contrast, in the TPL image, both the signal information and the background information are mixed together. Thus, the achieved contrast in the PT-OCT images proves that the PT-OCT method can be an alternative to TPL detection in imaging the photothermal trapping of GNRs, although PT-OCT has its shortcomings that need to be resolved. Specifically, in TPL imaging, the variation of the thickness of the aggregated GNRs can be connected to the variation of the magnitude of the TPL signal. In comparison, the PT-OCT image is not able to precisely present the thickness variation since it cannot convert the thickness information into the brightness variation in the image without the application of a reliable phase-unwrapping algorithm which is the target of the future work.

Finally, the fluctuation of the FFT signal and the varying pattern in the en-face images indicate that the photothermal trapping induced migration of GNRs in biological tissue is influenced by the complex and particular nature of tissue structure, and the photothermal trapping of GNRs in tissue has a greater complexity than in the DI water.

\section{Acknowledgements}

The authors thank Dr. Adrian Bradu and Dr. Manuel Jorge Marques for useful discussions and sharing previously designed LabVIEW code. Their valuable suggestions on the synchronization of galvo-scanners and data acquisition provided a practical reference for the corresponding author to write the LabVIEW programs used in this study. The authors also appreciate the valuable advice on data processing received from Dr. Sylvain Rivet. Yong Hu acknowledges the support received from the University of Kent. Adrian Podoleanu is also supported by the NIHR Biomedical Research Centre at Moorfields Eye Hospital NHS Foundation Trust and the UCL Institute of Ophthalmology and the Royal Society Wolfson Research Merit Award. 